Research Article

\title{
Cloud Features of Tibetan Plateau Vortex Category Cloud Cluster over Different Regions along the Eastward-Moving Path in Summer
}

\author{
Chao Li $\mathbb{D},{ }^{1}$ Xiaofang Wang $\left(\mathbb{D},{ }^{1}\right.$ Lingli Zhou, ${ }^{1}$ Chunguang Cui, ${ }^{1}$ Xingwen Jiang, ${ }^{2}$ \\ and Guirong $\mathrm{Xu}{ }^{1}$ \\ ${ }^{1}$ Institute of Heavy Rain, China Meteorological Administration, Wuhan, China \\ ${ }^{2}$ Institute of Plateau Meteorology, China Meteorological Administration, Chengdu, China \\ Correspondence should be addressed to Xiaofang Wang; tiaotiao98@163.com
}

Received 10 November 2018; Revised 7 January 2019; Accepted 6 February 2019; Published 7 March 2019

Academic Editor: Harry D. Kambezidis

Copyright (C) 2019 Chao Li et al. This is an open access article distributed under the Creative Commons Attribution License, which permits unrestricted use, distribution, and reproduction in any medium, provided the original work is properly cited.

\begin{abstract}
Using the data of CloudSat satellite, FY series satellite, CMORPH hourly precipitation, and ERA-interim reanalysis products, this paper aims to reveal the cloud features of Tibetan Plateau Vortex (TPV) category cloud clusters over its eastward-moving regions. 107 cases of eastward-moving TPV category that occurred in the summer half-year (April to September) are picked out, and then the cloud features of them are further analyzed by statistics. The results show that the eastward-moving TPV category occurs mostly in May and June, but leastly in July and September. With consecutive enhancement of precipitation intensity and convection intensity, an increasing trend is found in the proportions of deep convection clouds and multiple layer clouds during the TPV category eastward movement. In order to reveal the inner connection among the precipitation intensity, the convection intensity, and the microphysical characteristics of TPV category cloud clusters, the TPV category cloud clusters are classified into different categories by the criteria of the precipitation intensity and the convection intensity separately. Consequently, the two different criteria share the commonality that the number concentration of both ice crystal and cloud droplets increases obviously with the enhancement of precipitation intensity or convection intensity. However, the discrepancy of conclusions also exists between the two classification criteria. A notable stretching upward trend is found in the number concentration distribution of the ice crystal and downward trend in the number concentration distribution of the cloud droplet. The same increasing trend is also discovered in the effective average radius of the ice crystal and cloud droplet. But the TPV category cloud clusters with severe convection do not present the similar variation trend both in the number concentration and the effective average radius. Hence, although the above findings confirm that the precipitation intensity, the convection intensity, and the distribution of cloud hydrometers are associated and interacting mutually, the closed function relationship among them cannot be established, and other meteorology factors related to the ambient conditions should also be taken into consideration as a complete cloud microphysical system.
\end{abstract}

\section{Introduction}

As known as the third pole on the Earth, the Tibetan Plateau plays an extremely important role in research on global climate change. A vast number of precipitation cloud clusters or cloud bands originate from the Tibetan Plateau or its surrounding areas, and these cloud clusters or cloud bands, as well as their radiation effects, not only change the Tibetan Plateau's local weather conditions and climate pattern [1] but also exert a profound influence on the weather and climate in the downstream of the Tibetan Plateau when they are accompanied by an eastward-moving weather system [2]. It has been confirmed that the majority of Tibetan Plateau Vortexes (TPV), acting as a kind of typical weather systems that generate in complex terrain on TP, are born in the central and western areas of the Tibetan Plateau, and finally they vanish in the east of the Tibetan Plateau in summer [3], while the minority of TPV continues to develop 
and move outward from the Tibetan Plateau, and consequently, mesoscale convective precipitation cloud clusters attached by these eastward-moving TPV then often trigger heavy storms at the middle and lower reaches of Yangtze River [4].

Previous study on the eastward-moving TPV cloud clusters mainly focus on the mechanism of TPV' generation and development, and as early as 1970s, Ye and Gao, the associated meteorologists, noted that, under favorable circulation conditions, a few cases of TPV could move out of the Tibetan Plateau to the east of China, causing disastrous weather events such as rainstorms and thunderstorms widely in the downstream area afterwards [5]. From then on, numerous meteorologists and forecasters continued to research on the mechanisms of the development and migration of TPV, as well as the mechanism that how TPV induce rainstorms. For example, Yu and Gao once pointed out that two major categories of influencing systems, namely, a low trough category and a wind shear category accompanied with eastward-moving TPV, which determines the development and migration of TPV [6]. When TPV moves out of the Tibetan Plateau, the major influencing weather system consisting of "trough in the north and vortex in the south" may trigger heavy rainstorms in northwest China. Moreover, when eastward-moving TPV are intruded by cold air on the ground, regional rainstorms are triggered in Sichuan Province [2]. Other view point is also proposed by Zhang et al. When TPV move outward from the main body of the Tibetan Plateau in the summer time, Southwest Vortex (SV) is sometimes triggered in the lower level of troposphere under favorable topographical conditions in the west of Sichuan Province; meanwhile, these Southwest Vortexes continue to develop and move eastward and subsequently interact with the Meiyu front system on the middle and lower reaches of the Yangtze River and trigger persistent rainstorms therein [7].

When time comes to the beginning of the 21 century, with further enrichment of satellite and radar data, research focus was shifted to analyze the feature of clouds accompanied the by eastward-moving TPV. In particular, scientists began to conduct investigation into the mechanisms that how the eastward-moving Tibetan Plateau cloud clusters trigger disastrous weather in the downstream area based on the analysis of the cloud microscopic structure. For example, Shi et al. counted the frequency and analyzed the characteristics of the activities of mesoscale convective cloud clusters within the Yangtze River basin and discovered a close relationship between the large floods that occur in the Yangtze River basin and more than 300 meso- $\alpha$ and meso- $\beta$ convective clouds originating from Tibetan Plateau [8]. Zhou et al. studied the feature of the ambient instability influenced by the eastward-moving Tibetan Plateau convective cloud clusters, and the research revealed that the vertical structure is composed of the airflow divergence in the higher layer and the airflow convergence in the lower layer, the high-low-level jets acting as the guiding roles, the abundant moisture conditions within the Yangtze River basin, and the instability of the atmospheric layer, which are prerequisites for the formation of the eastward movement of convective cloud clusters over the Tibetan Plateau [9]. Recently, $\mathrm{Wu}$ revealed the evolution characteristics of cloud clusters accompanied by eastward-moving TPV, based on millimeter wave radar observation data, showing that the horizontal structure the TPV embody as a major precipitation band and several precipitation cloud clusters, while a spiral cloud band becomes incorporated into the center of the vortex from the south [10]. In addition, mesoscale convective systems (MCSs) exhibit fluctuating distributions with eastward transmission along $30^{\circ} \mathrm{N}$ in China, according to an analysis of the movement and transmission characteristics of MCSs by Zheng et al. [11].

CloudSat is a polar orbit satellite launched by NASA in 2006, aiming at detecting the global distribution and vertical structural features of clouds. Because of the profile radar onboard, CloudSat has advantages in detecting the vertical structures of clouds, and thus its associated data products has been widely used to study macroscopic characteristics and hydrometers distribution in different categories of clouds in recent years. Thus, CloudSat data products are applied not only to analyze different categories of clouds in different regions under climatic statistics [12-15], but also to research on microstructure features of different categories of clouds for specified weather systems $[16,17]$.

Although previous research relevant to the eastwardmoving TPV and its accessory clouds originated from the Tibetan Plateau has acquired a great deal of highly significant discoveries, there are still many deficiencies. Due to the absence of observation and study on the distribution of a hydrometer in these clouds and the characteristics of evolution, it leads to incomprehension in the mechanism of how these clouds trigger heavy rainstorm. As a result, the ability to predict these extreme weather events inevitably remains at a relatively low level. Accounting for all the reasons above, this paper attempts to reveal the cloud feature of the eastward-moving TPV cloud clusters by comparing the difference of TPV cloud clusters over different regions along the eastward-moving path, based on CloudSat products. This paper adopts the research route above mainly taking the following factors into consideration. First, CloudSat has an excellent ability to detect the vertical structure of clouds, so it is advantageous for scientists to discover much more microstructural feature with regard to different categories of clouds. Second, hitherto, CloudSat data product was applied in the climatic statistical analysis and the analysis of microstructural feature of clouds for specified weather system separately, and this paper attempts to combine the two aspects above together, namely, adapting the method that integrates the statistical analysis with diagnoses of cloud microphysics to research the TPV cloud clusters. However, CloudSat, acting as a global polar orbiting satellite, rather than geostationary satellite, cannot provide consecutive observation for the eastward-moving TPV cloud clusters in a specified area. So this paper further optimizes the research route. First, the eastward-moving path was divided into three different key regions based on topography differences, namely, the Tibetan Plateau, the eastern slope of the Tibetan Plateau and its surrounding areas, and the middle and lower reaches of Yangtze River. Then, the cloud feature was 
analyzed and compared by the statistical results that originated from the three different key regions.

\section{Initial Data and Methodology}

2.1. Initial Data. The initial datum applied in this paper mainly contains four categories of data, which consist of European Center for Medium-Range Weather Forecasts (ECMWF-Interim), Six hourly global reanalysis data (including geopotential field, humidity field, and wind field with a horizontal resolution of 0.75 degrees $\times 0.75$ degrees), CloudSat $2 \mathrm{~B}$ series and $2 \mathrm{C}$ series data products (including 2B-CWC-RO, 2B-GEOPROF, 2B-CLDCLASS, and 2C-PRECIPITATION), temperature of black body (TBB) data inversed from the FY (Fengyun) stationary satellite series, and climate prediction center morphing technique (CMORPH) hourly fusion precipitation data from the National Meteorological Information Center. Then, the valuable information is extracted from initial data to carry out further research through the following methodology.

\subsection{Definition of TPV Category and Selection Criteria.} First, the eastward-moving TPV category cases that would reach the eastern coast of China were picked out based on the 4 times daily circulation field in the summer half-year (i.e., from April to September) during both 2007 and 2016. The selection criteria were as follows: (1) a closed cyclonic circulation presented within geopotential field or wind field at the $500 \mathrm{hPa}$ isobaric surface (the circulation circle was closed or exceeded $3 / 4$ of a circle within the prescribed area at the geopotential height field; the cyclonic circulation was presented in wind field), and the diameter of the circulation satisfied the criteria of a meso- $\alpha$ scale vortex (i.e., the length of the short axis diameter ranged from $10^{2} \mathrm{~km} \sim 10^{3} \mathrm{~km}$ ). (2) A part of nascent TPV was moved outward from the Tibetan Plateau and continued to develop and move eastward, until arriving at the lower reaches of the Yangtze River in the east direction. (3) For the circulation pattern of the TPV that moved outward from the Tibetan Plateau, either the closed cyclonic circulation continued to persist at the $500 \mathrm{hPa}$ isobaric surface, or the closed cyclonic circulation evolved into a deep trough but lead the formation of Southwest Vortex (SV) at the $700 \mathrm{hPa}$ isobaric surface, and the latter weather system configuration was similar with the coupling form between TPV and SV proposed in [18], which could also migrate to the lower reaches of the Yangtze River in the east direction, and subsequently may triggered heavy rainstorm in the downstream area as the former one. Therefore, these two different weather processes above were integrated into a whole group called the eastwardmoving TPV category in this paper.

The cloud clusters affiliated with the eastward-moving TPV category move to the lower reaches of the Yangtze River along three different paths (i.e., the northeastward migration path, the eastward migration path, and the southeastward migration path) [3]. But this paper only researched the eastward-moving TPV category that moved along the eastward migration path, which trigger a series of heavy precipitation in the Yangtze River basin. The eastward migration path could be divided into three different key regions listed above, namely, the Tibetan Plateau region, the eastern slope of the Tibetan Plateau and its peripheral region, and the middle and lower reaches of the Yangtze River region. The scope of longitude and latitude for the three different regions above was set as the following (Figure 1): the scope of the Tibetan Plateau region was limited to the rectangular area of $83^{\circ}-100^{\circ} \mathrm{E}$ and $27^{\circ}-40^{\circ} \mathrm{N}$ (represented by $\mathrm{A} 1$, short for TP), the scope of the eastern slope of the Tibetan Plateau and its peripheral region was limited to the rectangular area of $101^{\circ}-110^{\circ} \mathrm{E}$ and $25^{\circ}-37^{\circ} \mathrm{N}$ (represented by A2, short for ESTP), and the scope of the middle and lower reaches of the Yangtze River region was limited to the rectangular area of $111^{\circ}-120^{\circ} \mathrm{E}$ and $25^{\circ}-35^{\circ} \mathrm{N}$ (represented by A3, short for MYR). Considering that the CloudSat is a polar orbiting satellite rather than a stationary satellite, the eastward migration of the TPV category is a consecutive weather process, so the effective observation window is crucial for the detection of the cloud clusters affiliated with the TPV category. Hence, the criteria of the effective observation window were set as the following: first, the time when an eastward-moving TPV category entered each key area was set as the benchmark time. Then, accounting for the translation speed of eastward-moving TPV category and the scope of cloud clusters affiliated to it, the effective period was windowed to six hours before or after the benchmark time. Next, whether the CloudSat star trajectory is passing through each key region within an effective observation window had been validated. If it was confirmed, the CloudSat data products within it was considered to be the effective detection to eastward-moving TPV category cloud clusters; otherwise, it was treated as ineffective. Finally, the whole effective observation data were collected through the procedure above.

2.3. Verification of CloudSat Data Accuracy. Owing to the excellent ability of the CloudSat to detect the vertical microstructure of clouds, it has been widely applied to research in different clouds. However, the credibility of CloudSat data has always been a concern for scientists. Barker et al. examined the discrepancy between CloudSat data and aircraft platform-based observation data for a series of the evolution process of cloud systems in the southern part of Quebec Province, Canada. The results show that the cloud extinction coefficient data inverted by CloudSat are reliable in the upper troposphere. Liquid cloud content and ice cloud content data are more reliable in the lower troposphere [19]. Qiu et al. investigated the discrepancy in cloud microphysical characteristics of mixed-phase cloud layers between weakly convective clouds and stratus clouds, detected by both CloudSat and aircraft, and the study reveals that the two categories of clouds have the same variation tendency with regard to the cloud microphysical characteristics. Although $20 \%$ bias of value existed between these two platforms, CloudSat observation data are still effective for 


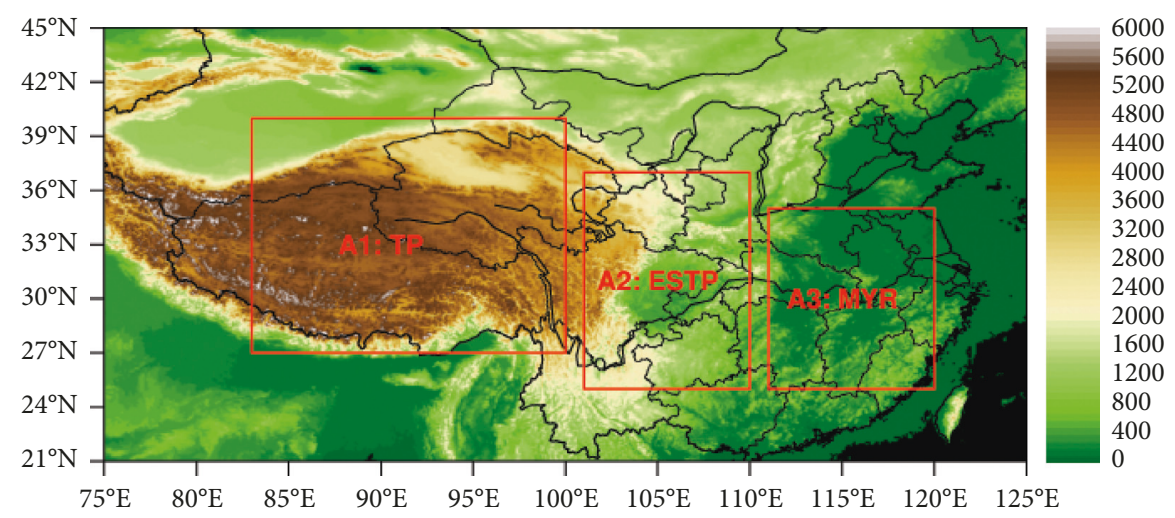

Figure 1: The location of the three key regions along the eastward-moving path (A1 represents the Tibetan Plateau (TP) region, A2 represents the eastern slope of Tibetan Plateau and its peripheral region (ESTP), and A3 represents the middle and lower reaches of the Yangtze River region (MYR)).

research on cloud microphysical characteristics. According to the conclusions above, it is possible to apply CloudSat data to analyze the cloud features of the eastward-moving TPV category cloud clusters [20].

\section{Results}

3.1. The Variation of Frequency, Precipitation, and TBB with regard to the Eastward-Moving TPV Category. 107 cases of the eastward-moving TPV category that moved in the east direction were picked out totally during the summer halfyear (i.e., from April to September) from 2007 to 2016, based on the selection criteria that was set in Section 2.2. To examine whether the statistical results gathered herein were exact or not, the statistical results in this paper were made contrast with the ones reported in [21, 22]. Feng et al. once discovered that approximately 8.7 cases of TPV move outward from Tibetan Plateau on annual average from 2000 to 2009 by statistics [21]. And Zhong et al. pointed out that approximately 1 case of CSW migrate eastward annually from 1979 to 2008 by statistics [22]. It means that the frequency of the TPV category picked out in this paper is approximately equal to the summation of the frequency of the eastward-moving TPV and SV selected by Feng and Zhong on annual average. Therefore, the statistical results in this paper are reliable.

The variation of eastward-moving TPV category is shown in Figure 2. The frequency of the eastward-moving TPV reaches a maximum during May and June, compared with a minimum during July and September. Obviously, it demonstrates a tendency of ascending first and descending later, and this conclusion is consistent with the statistical results drawn by $\mathrm{Yu}$ and Gao [3]. In addition, the circulation factors including both the interaction between westerly belt synoptic system, subtropical synoptic system, and tropical synoptic system in the midlevel of troposphere and the coordination of divergence of wind, the front zone, and the downward transmission of potential vorticity in the upper level of the troposphere play a dominant role of influencing the generation and development of TPV. However, the condition of the circulation factors mentioned above will

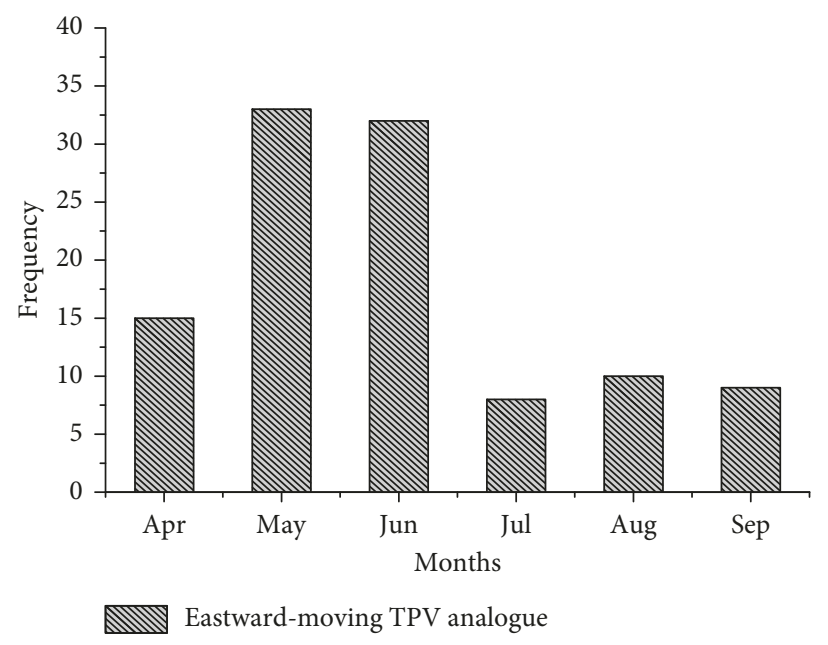

FIGURE 2: The monthly variation of the frequency with regard to the eastward-moving TPV category ( $X$-axis represents the month, and $Y$-axis represents vortex frequency (unit: case)).

change with the alternation of month, and thus the advantageous circulation field dominates the monthly variation trend $[23,24]$. As shown in Figure 3, the intensity of the acreage-average precipitation triggered by the eastwardmoving TPV category successively strengthens when the eastward-moving TPV category passes through each key region. And as shown in Figure 4, the TBB on cloudtop of the TPV category cloud clusters is mainly distributed in the scope of negative 60 degrees centigrade to negative 45 degree centigrade. The relatively high value (i.e., value above negative 65 degree centigrade) of TBB approximately shows an descending tendency, meanwhile the relatively low value (i.e., value below negative 65 degree centigrade) of TBB approximately shows an ascending tendency. The variation discipline indicates that the height of cloudtop may elevate with regard to the TPV category cloud clusters or the convection intensity within them enhanced during their eastward migration. Generally speaking, the statistical results of basic feature with regard to the TPV category in this paper are consistent with the conclusion drawn in the previous reference. 


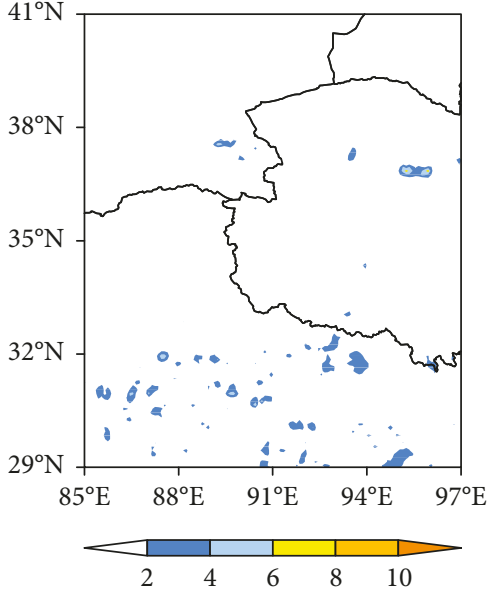

(a)

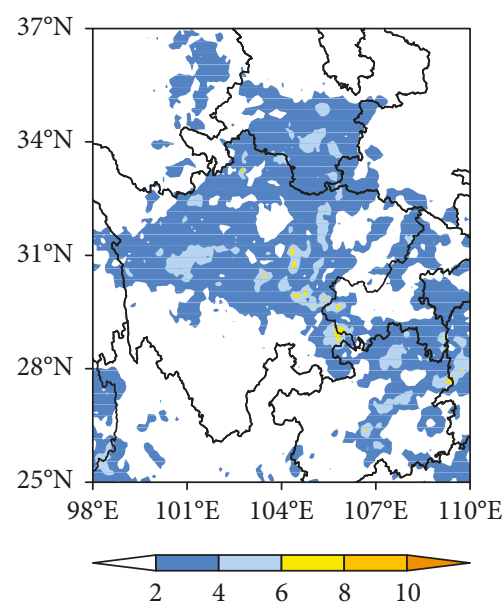

(b)

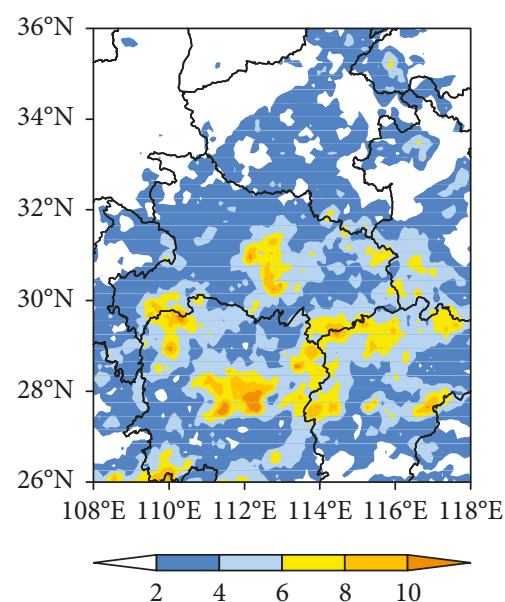

(c)

FIGURE 3: The distribution of the area-average precipitation triggered by the eastward-moving TPV category over different key regions: (a) TP; (b) ESTP; (c) MYR (shaded color represents precipitation (unit: $\mathrm{mm}$ )).
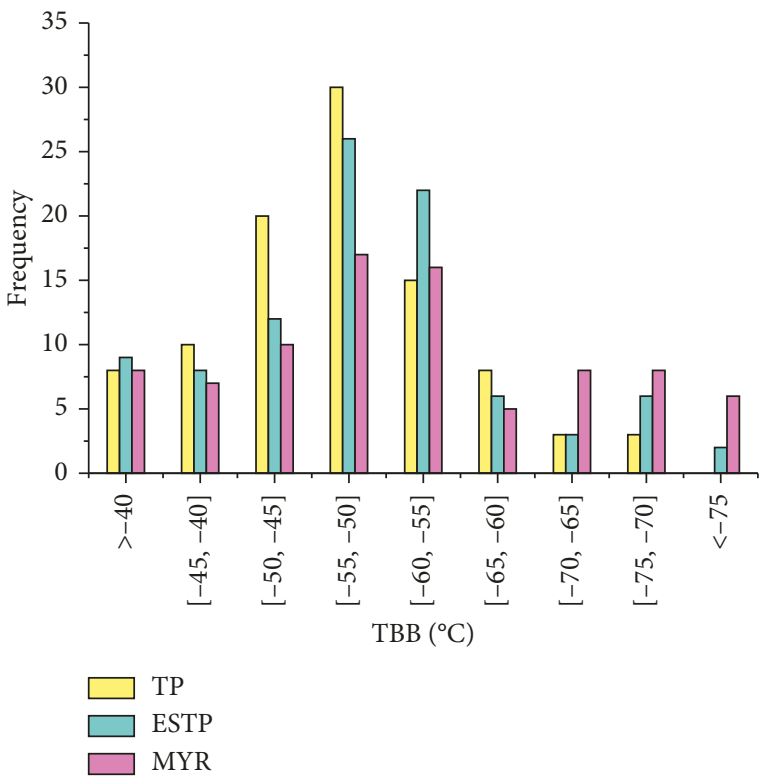

FIgURE 4: The distribution of TBB on cloudtop with regard to the eastward-moving TPV category cloud clusters over different key regions (light yellow represents $\mathrm{TP}$, light green represents ESTP, light red represents MYR, the $X$-axis represents TBB (unit: ${ }^{\circ} \mathrm{C}$ ), and the $Y$-axis represents frequency (unit: case)).

\subsection{Comparison of Cloud Features of the TPV Category over} Each Key Region. Enormous differences of the cloud classification exist among each key region with regard to TPV cloud clusters. As shown in Figure 5, the most significant difference of the statistical results between the TPV category cloud clusters and all categories of clouds under climatological background state lies in the proportion of deep convection clouds at different high levels, especially in the low level. It is owing to the diversity between TPV category and other eastward-moving synoptic systems initiated from Tibetan Plateau under the climatological background state, the eastward-moving TPV category possesses the deep updraft structure with intense convergence in the midlow level $(500 \mathrm{hPa})$ and intense divergence in the upper level $(200 \mathrm{hPa})$, and thus it leads the preservation of deep convection. Furthermore, the consecutively enhancing precipitation triggered by TPV category cloud clusters ensures the reinforcement of latent heat release, and the thermal energy generated by that is crucial for the preservation of deep thermal-forcing updraft and then leads the promotion of convergence in the lower level and divergence in the upper level consequently. Thus, the above advantageous ambient field is likely to trigger more deep convection practically and inevitably the proportion of deep convection clouds for the TPV category exceeding that one under the climatological background state. In addition, nimbostratus clouds (i.e., NS) proportion in the statistical results of the TPV category cloud clusters is fewer than that one under the climatological background state over the TP region, but more in the downstream area of TP (i.e., the ESTP and MYR region), and stratocumulus clouds (i.e., ST) proportion in the TPV category cloud clusters is fewer than that one under the climatological background state at the lower level, but more in the middle and higher level in the downstream area of TP (i.e., the ESTP and MYR region). Besides, the proportion of deep convection clouds and other stratus clouds over the three different key regions is also obviously different, namely, the proportion of convection clouds almost presents an ascending tendency, while the proportion of stratus clouds almost presents an ascending tendency during the eastward movement of the TPV category. Although the statistical results of both the TPV category cluster clouds and all categories of clouds under climatic state shared some similarities among the three different key regions, the severe convection feature is more outstanding in the TPV category cloud clusters, rather than other clouds. With further analysis of cloud thickness and precipitation cloud attributes (as shown in Figure 6), more cloud features are revealed, the proportion of single-layer clouds in TPV category cloud clusters is less than that one under the climatic state, and 


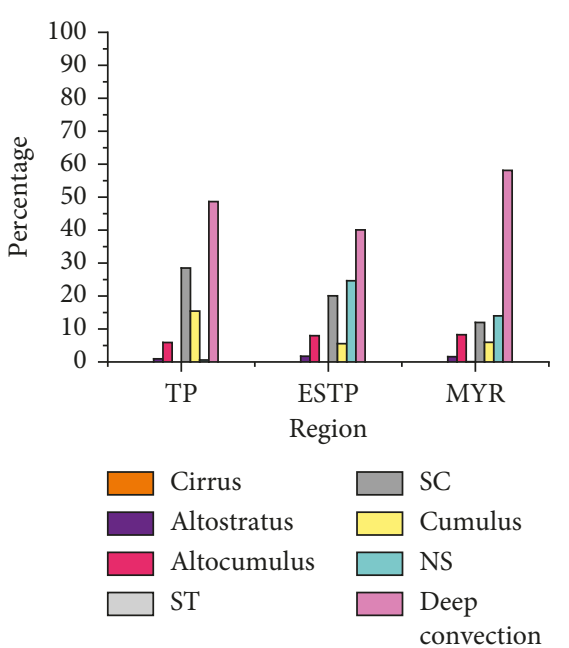

(a)

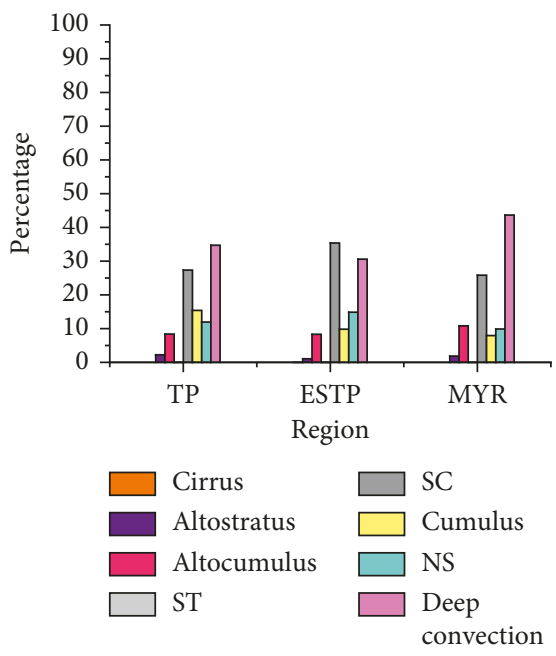

(d)

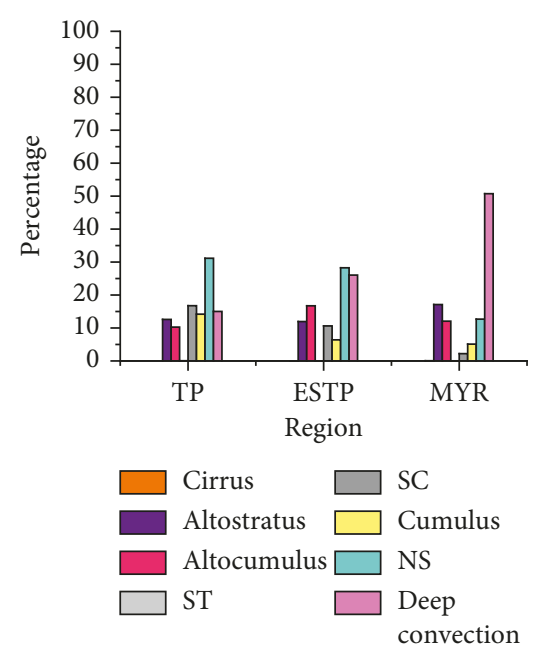

(b)

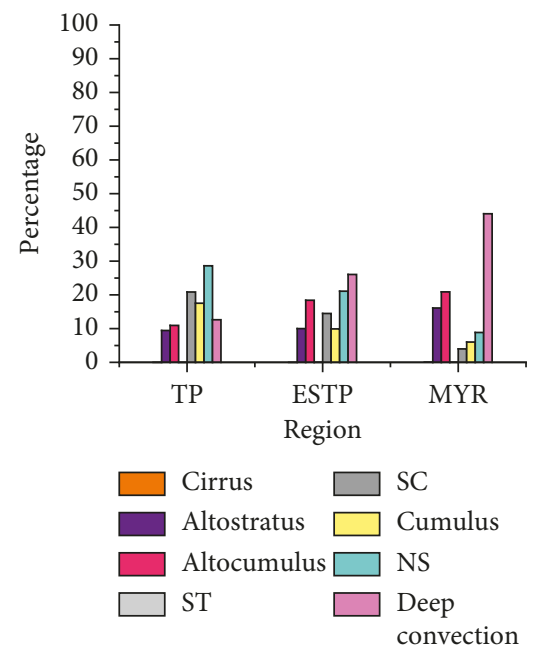

(e)

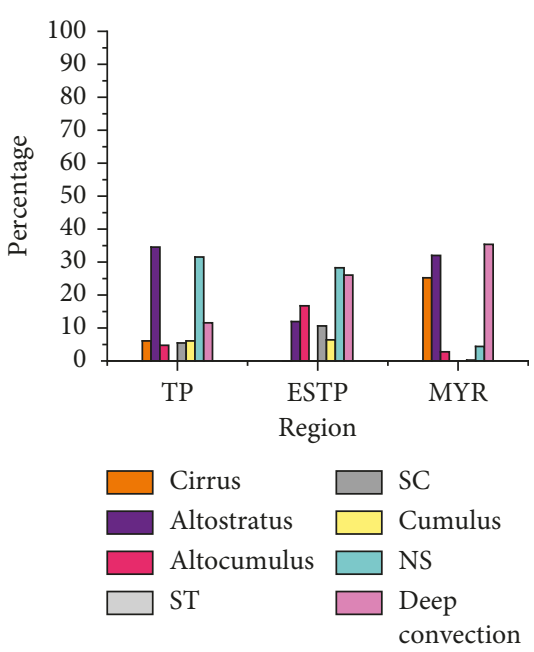

(c)

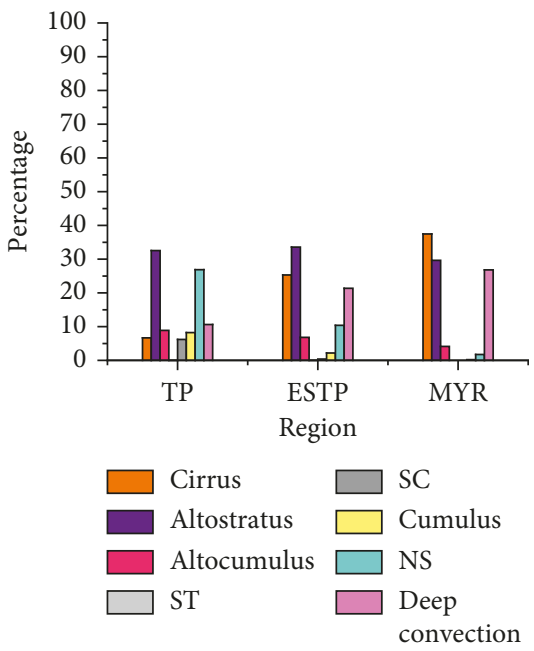

(f)

Figure 5: The statistical results of the clouds classification at different altitude levels over each key region. TPV clouds in the (a) lower level (1-5 km), (b) middle level (5-10 km), and (c) higher level (10-15 km).Climatological clouds in the (d) lower level, (e) middle level, and (f) higher level ( $X$-axis represents different key regions; and $Y$-axis represents percentage of frequency (unit: \%)).

correspondingly, the proportions of double layers and multiple layers clouds in TPV category cloud clusters are more than those ones under the climatic state. Moreover, the proportion of convective precipitation clouds in the TPV category cloud clusters is more than all categories of clouds under the climatic statistics, and correspondingly, the proportion of shallow precipitation clouds in them is less. The ascending tendency of cloud layer quantity and the enhancement of convection precipitation are presented during the eastward movement of TPV category cloud clusters. Although the statistical results under climatological background state share a part of similar discipline in the aspects of layer quantity and convection precipitation attributes over the three key regions, these two attributes in TPV category cloud clusters are more outstanding. Analyzing the causes for variation tendency shown in Figure 6, it is revealed that the water vapor transportation and the local atmosphere circulation are the key factors; that is to say, when the activity of eastward-moving TPV category cloud clusters is limited within the TP area, the supply of water vapor transportation for satisfying the further development of cloud clusters is in shortage. However, when TPV category cloud clusters enters the ESTP area, the abundant water vapor transportation carried by the westerly jet of the Tibetan Plateau southern branch adds the supply, and thus the development of cloud clusters is boosted. In addition, the favorable topography in the leeward side of Tibetan Plateau as well as the enhancement of vertical flow supported by the upper level trough or vortex promote the convergence of wind in the lower level, and all these above factors result in the increase of cloud layer quantity and the enhancement of convection precipitation over the ESTP area accordingly. Finally, when TPV category cloud clusters enters the MYR area, excepting the more adequate supply of water vapor transportation carried by the low-level southwest jet along the western edge of subtropic high, the cyclonic low-level convergence located in the left side of the jet is advantageous for the further development of cloud clusters, and these 


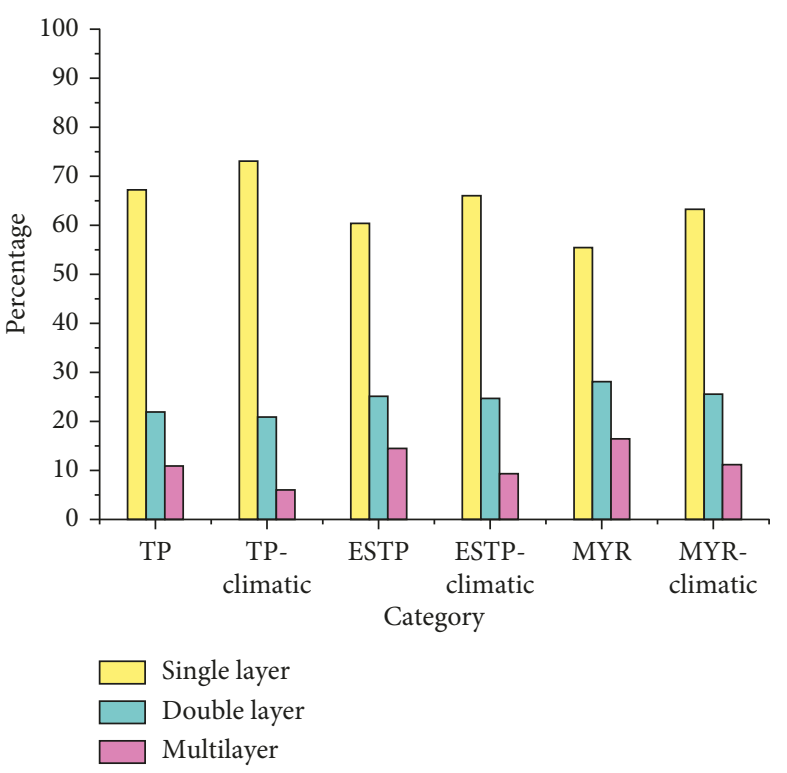

(a)

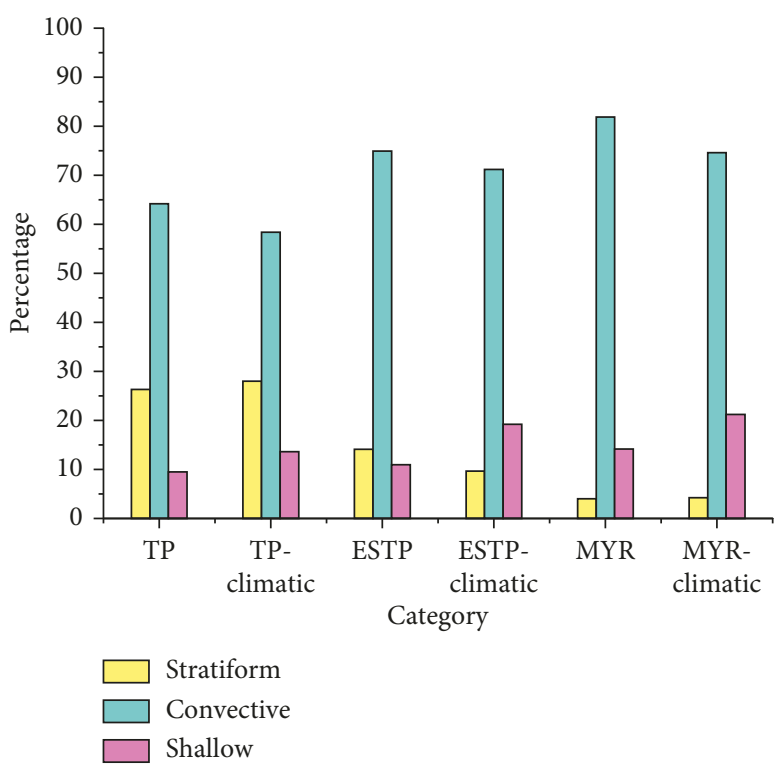

(b)

FIGURE 6: The statistical results of cloud layer quantity and cloud precipitational attribute over each key region ((a) cloud layer quantity; (b) precipitational cloud attribute for vertical wind motion. $X$-axis represents different key areas, and $Y$-axis represents percentage of frequency (unit: \%)).

above factors ensure the consecutive increase of cloud layer quantity and the enhancement of convection precipitation over the MYR area. Anyhow, the statistical results above are approximately consistent with the ones in Gao's dissertation focusing on the macroscopic characteristics of precipitation cloud bands located in the same latitudinal zone with Tibetan Plateau [25].

Making further research on the distribution of cloud hydrometer particles in the eastward-moving TPV category cloud clusters is essential to make deeper comprehension of microstructure of them (as shown in Figure 7), it revealed that the average effective radius of ice crystal particles for the TPV category cloud clusters is more than all categories of clouds under the climatic state within the main distribution level $(6 \mathrm{~km}-12 \mathrm{~km}$ in the troposphere) in each key regions, and the average effective radius of ice crystal particles increases gradually during the eastward movement (Figure 7(a)). The variation of them is closely related to the enhanced convective precipitation properties and vertical development of cloud clusters. However, the average effective radius of water droplet particles for the TPV category cloud clusters is almost less than all categories of clouds under the climatological background state in each key regions, and the average effective radius of water droplet particles within the main distribution height interval for the TPV category cloud clusters decreases gradually, especially above the level of $3 \mathrm{~km}$ (Figure 7(b)), which is opposite to the ice crystal particles. As is known to all, the strengthening of vertical wind shear and updraft triggered by the convection is advantageous to the fragmentation of cloud droplets and thus disadvantageous to the increase of the average effective radius for cloud droplets during the eastward movement of TPV category. Guo et al. once pointed out the mechanism that the weather conditions affecting the variation of cloud microparticles is complex, mainly including the difference of the precipitation efficiency, the convection intensity, the topography effect, the water vapor transportation, and the interaction among them [26].

In brief, the statistical results of cloud hydrometer particles in both TPV category cloud clusters and all clouds under the climatological background state have some similarities likewise, but the former ones are more outstanding. It is connected to the macroscopic feature of clouds in microscopic perspective. The eastward-moving TPV category cloud clusters as a kind of a typical mesoscale convective system is clearly distinct from other clouds under the climatological background state in the precipitation efficiency and the convection intensity mentioned in Section 3.1. Considering that the variation of the cloud hydrometer and macroscopic cloud feature is closely correlated with each other, the precipitation efficiency, the convection intensity, and the topography effect are probably the dominated factor to affect the evolution of the TPV category cloud cluster, and thus it is essential to carry out more investigation on relationship among them.

\subsection{Comparison between Different Types of TPV Category} Cloud Clusters Based on Difference in Precipitation Intensity. The difference in the efficiency of transformation between the cloud hydrometers and rainwater is significant [27]. Therefore, it is necessary to make a contrast among different TPV accompanied with various precipitation intensities. And it will be contributive to reveal the inner connection between TPV category cloud clusters and precipitation efficiency. 


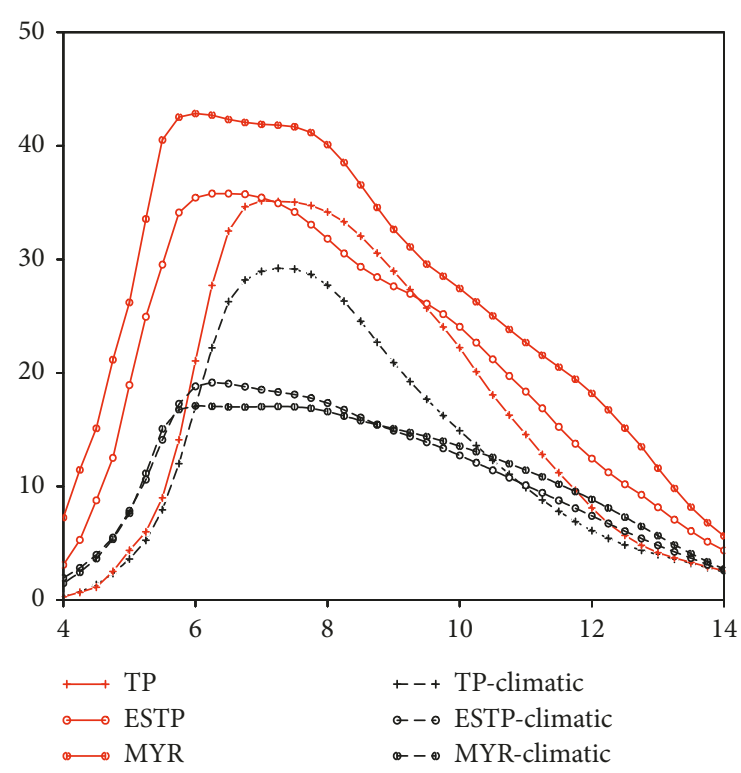

(a)

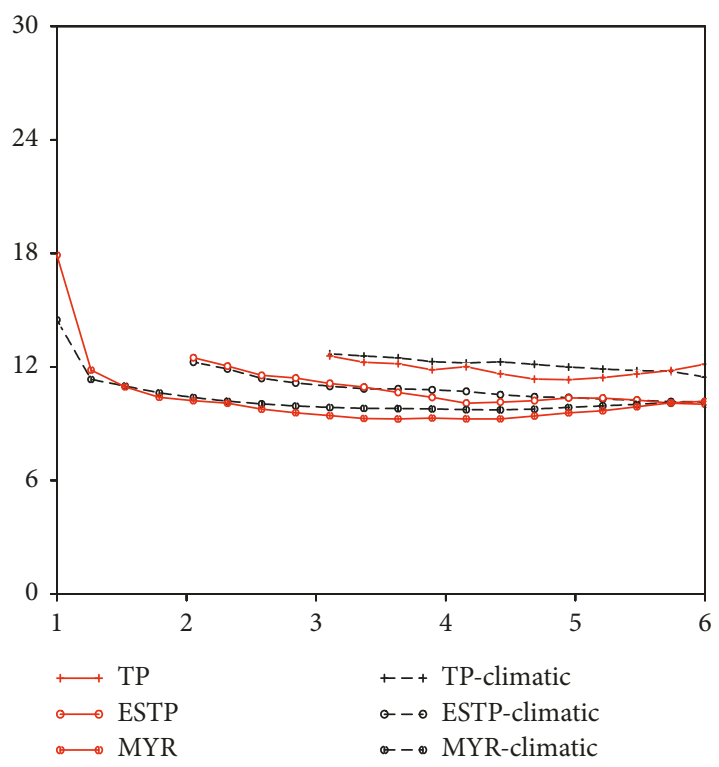

(b)

FIGURE 7: The statistical results of average effective radius of hydrometers within TPV category cloud clusters over each key region: (a) effective radius of ice crystal particles; (b) effective radius of water-droplet particles (black legend represents climatological results, $X$-axis represents altitude (unit: $\mathrm{km}$ ), and $Y$-axis represents average effective radius (unit: $\mu \mathrm{m}$ )).

First, the selected cases of TPV category cloud clusters were classified into two groups based on the difference in the precipitation intensity. The China Meteorological Administration (CMA) has only issued the precipitation intensity classification standard based on 12 and 24 hours cumulative precipitation, but the standard do not involve hourly cumulative precipitation classification criterion. Accounting for the transient variation characteristics of cloud hydrometers, the classification criterion of hourly cumulative precipitation is more appropriate to reflect the interrelation between TPV category cloud clusters and precipitation efficiency; hence, this paper adopts the similar classification criterion of hourly cumulative precipitation proposed by Gao [25] in his doctoral dissertation. Gao classified the clouds into three groups based on the difference of hourly cumulative precipitation as follows: (1) rainless type (area and hourly average precipitation $=0 \mathrm{~mm}$ ), (2) light rainfall type $(0 \mathrm{~mm}<$ area and hourly average precipitation $\leq 1 \mathrm{~mm})$, and (3) heavy rainfall type (area and hourly average precipitation $>1 \mathrm{~mm}$ ). Considering that the eastward-moving TPV category cloud clusters could bring precipitation in most cases, this paper adjusted the classification criterion above as follows (as shown in Table 1): (1) light rainfall type $(0 \mathrm{~mm}<$ area and hourly average precipitation $\leq 1 \mathrm{~mm})$ and (2) heavy rainfall (area and hourly average precipitation $>1 \mathrm{~mm}$ ). Then, the vertical distribution of hydrometers is made contrast between the two groups by statistical results.

The number concentration and the average effective radius of hydrometers is an important index to reflect the microstructure of clouds. As shown in Figure 8, the number concentration of ice crystals at the medium of the troposphere increases with the enhancement of precipitation intensity (i.e., the "heavy rainfall" type compared with the
TABle 1: The classification criteria of TPV category cloud clusters based on the difference of precipitation intensity.

\begin{tabular}{lc}
\hline Classification criterion & $\begin{array}{c}\text { Regional mean hourly } \\
\text { precipitation }(\mathrm{RMHP})\end{array}$ \\
\hline Light rainfall type & $0 \mathrm{~mm} / \mathrm{hr} \leq \mathrm{RMHP} \leq 1 \mathrm{~mm} / \mathrm{hr}$ \\
Heavy rainfall type & $\mathrm{RMHP}>1 \mathrm{~mm} / \mathrm{hr}$ \\
\hline
\end{tabular}

"light rainfall" type) in each key region. Also, the ice crystals distributed near the tropopause $(12 \mathrm{~km}-15 \mathrm{~km})$ presented a tendency of stretching upward with the enhancement of precipitation intensity in each key region. Absolutely, the distribution of ice crystals in either "light rainfall" type or "heavy rainfall" type demonstrates a tendency of stretching upward in the higher level of troposphere and stretching downward in the lower level of troposphere during the TPV category eastward movement, and this variation discipline is in coincidence with the variation of TBB on cloudtop. In addition, the average effective radius of ice crystals likewise shows an ascending tendency in the middle and higher level of the troposphere $(7 \mathrm{~km}-16 \mathrm{~km})$, correspondingly by the contrast between the "heavy rainfall" type and "light rainfall" type (as shown in Figure 9(a)), and the average effective radius of ice crystals in the "heavy rainfall" type increases obviously in the downstream of TP.

The vertical distribution of cloud droplets is distinct from ice crystals, as shown in Figure 10, and the number concentration of cloud droplets increases in the middle and higher level of troposphere $(6 \mathrm{~km}-9 \mathrm{~km})$ when the precipitation intensity strengthens (i.e., the "heavy rainfall" type compared with the "light rainfall" type) in each key region. Considering that the ice crystals are also abundantly distributed in the height scope $(6 \mathrm{~km}-9 \mathrm{~km})$ where cloud 


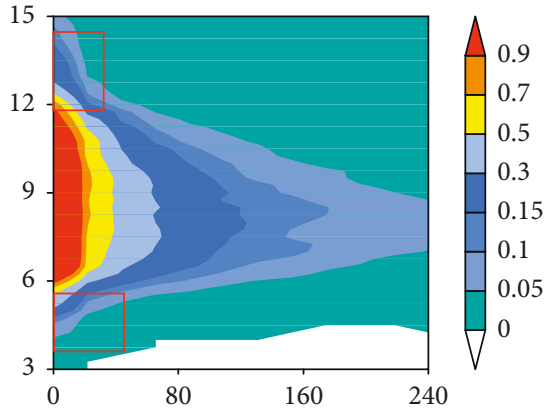

(a)

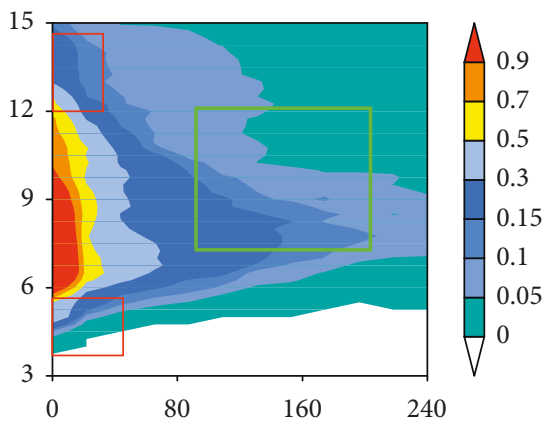

(d)

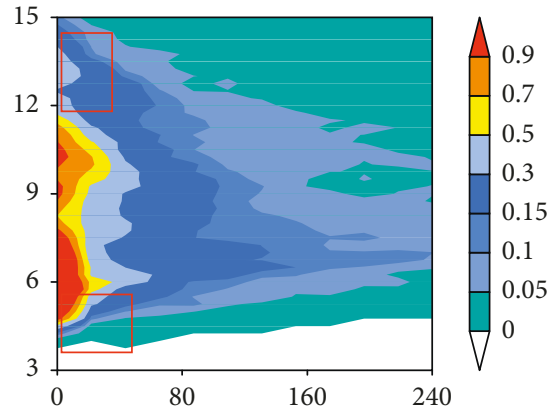

(b)

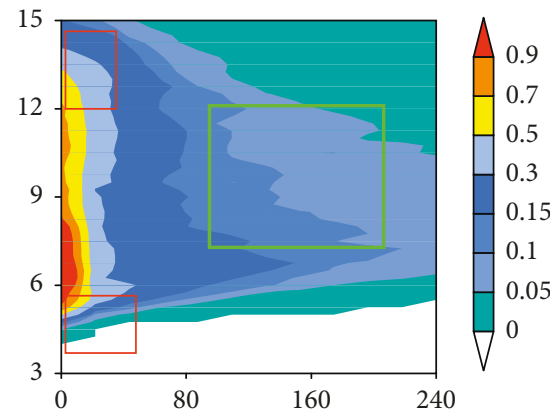

(e)

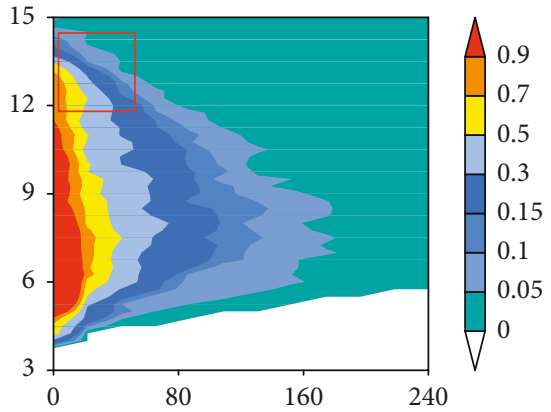

(c)

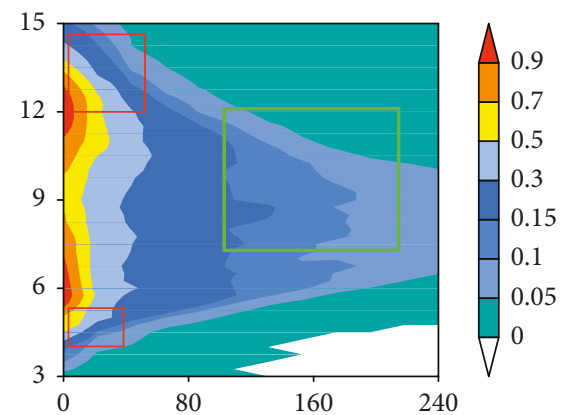

(f)

Figure 8: The vertical distribution of the number concentration of ice crystals corresponding to different types of TPV category cloud clusters over each key region: (a) iwc_TP (LR); (b) iwc_ESTP (LR); (c) iwc_MYR (LR); (d) iwc_TP (HR); (e) iwc_ESTP (HR); (f) iwc_MYR (HR) ((a) TP, (b) ESTP, and (c) MYR represent light rain type, and (d) TP, (e) ESTP, and (f) MYR represent heavy rain; $X$-axis represents particles number concentration (unit: $\mathrm{mg} / \mathrm{m}^{3}$ ); $Y$-axis represents altitude (unit: $\mathrm{km}$ ); shaded color represents the percentage of frequency (unit: \%); and red and yellow box represents the significant discrepancy area)).

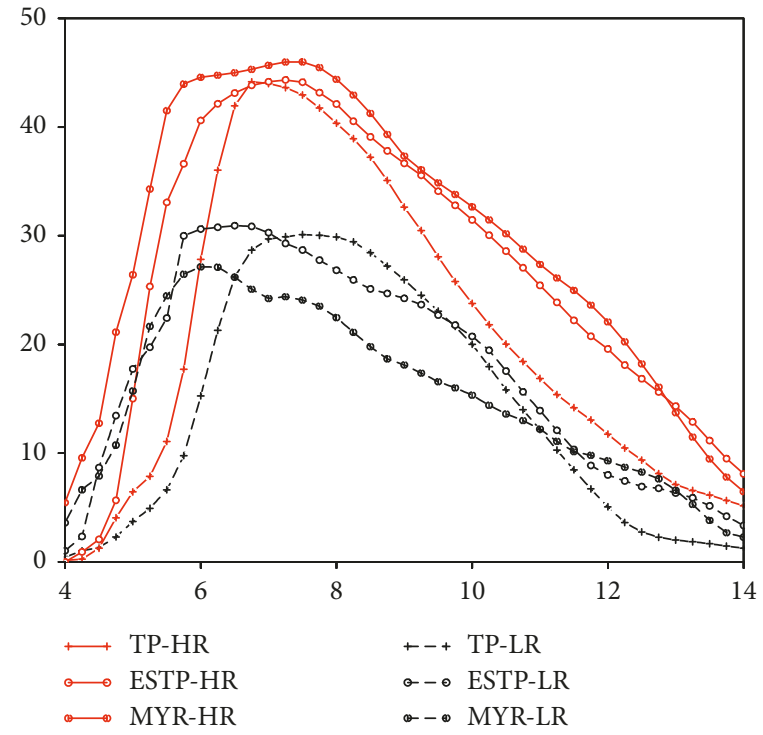

(a)

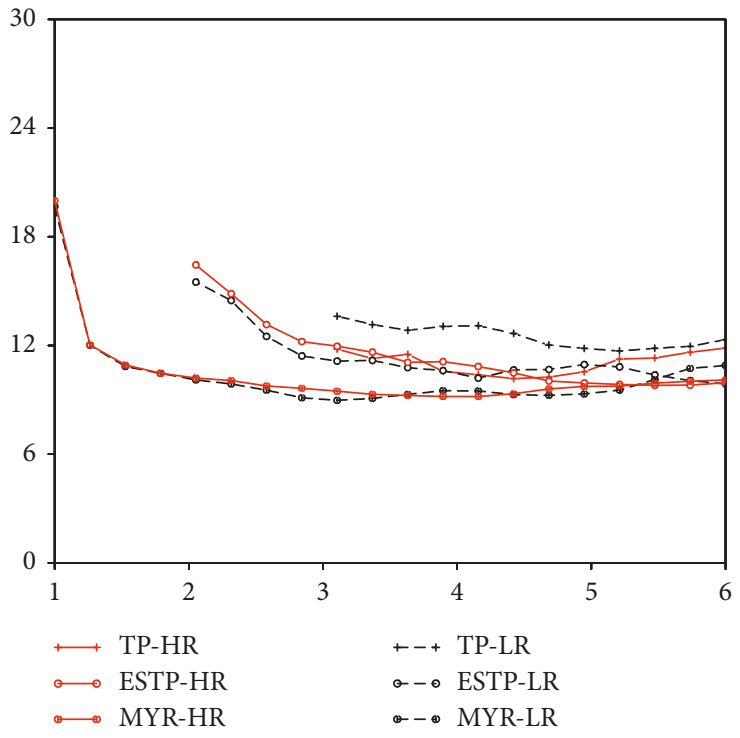

(b)

FIGURE 9: The vertical distribution of the average effective radius of different hydrometer particles corresponding to different types of TPV category cloud clusters in each key region: (a) effective radius of ice crystal particles; (b) effective radius of water-droplet particles ((a) ice crystals, (b) cloud droplets, red legend represents heavy rainfall type, the black legend represents light rainfall type, $X$-axis represents altitude (unit: $\mathrm{km}$ ), and $Y$-axis represents average effective radius (unit: $\mu \mathrm{m}$ )). 


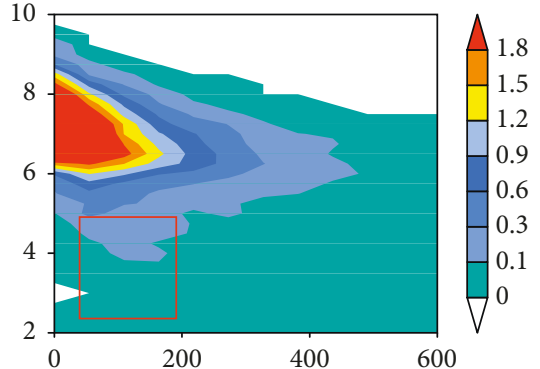

(a)

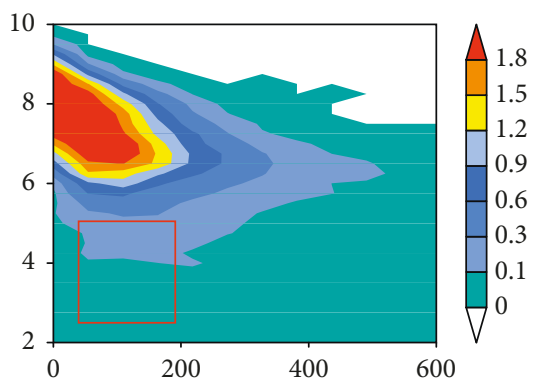

(d)

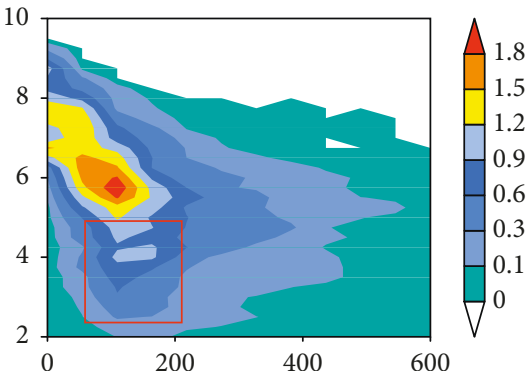

(b)

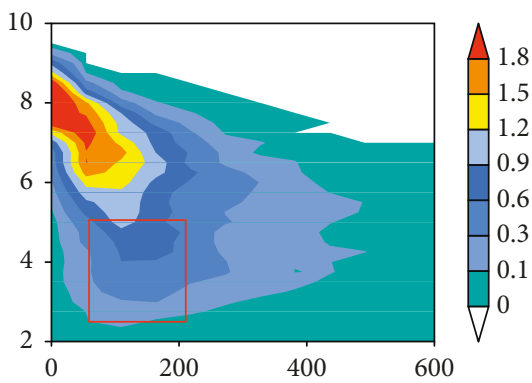

(e)

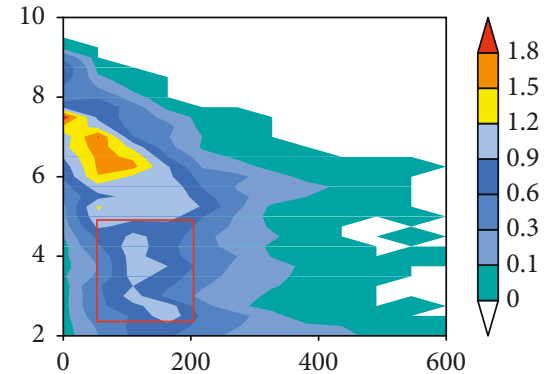

(c)

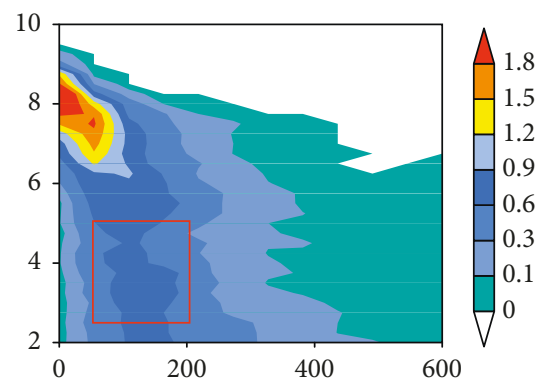

(f)

FIGURE 10: The vertical distribution of the number concentration of cloud droplets corresponding to different types of TPV category cloud clusters over each key region (same as above): (a) lwc_TP (LR); (b) lwc_ESTP (LR); (c) lwc_MYR (LR); (d) lwc_TP (HR); (e) lwc_ESTP (HR); (f) lwc_MYR (HR).

droplets abundantly distributed as well, the liquid water is likely to exist in the form of supercooled water or ice-water mixed-phase. Thus, it can be concluded that the enhancement of the precipitation intensity is advantageous to the increase of the concentration of cloud droplets in the ice water mixed layer. Furthermore, the distribution of cloud droplets tends to stretch downward rather than upward during the TPV category eastward movement. The average effective radius of cloud droplets presents a coincident variation discipline with the number concentration, that is, the average effective radius of cloud droplets showing increasing tendency near the ground corresponding to each key region, and meanwhile, the decreasing tendency of average effective radius of cloud droplets is also obvious in midair (Figure 9(b)). It is likely that the fragment and collision of cloud droplets play a dominant role in the variation of the average effective radius.

In a word, although the conclusion drawn in this paper is consistent with Gao, this paper has made further supplement to reveal more details about TPV category cloud clusters, rather than common clouds located on the same latitude zone with TP. But beyond the previous findings [25], the above results confirm the inherent association between the hydrometeors (ice crystals and cloud droplets) and precipitation intensity as supplementary research. And the association is mainly embodied in the synchronous changes of the variation tendency. During the eastward movement of the TPV category, the intensity of average precipitation presents increasing tendency shown in Figure 3, while the number concentration of ice crystals presents the increasing tendency in middle and upper level correspondingly, as shown in Figure 8, and the water droplets presents increasing tendency in the lower level, as shown in Figure 10 likewise. Moreover, previous study [27] has suggested that the increase of number concentration concerning ice crystals and water droplets may result from the reinforcement of icy cloud microphysical process and melting microphysical process separately, and these two microphysical processes are also a part of significant factors to dominate the variation of precipitation intensity [28]. Therefore, the attributes of hydrometeors are closely related with the precipitation intensity indeed. Although precipitation intensity is one of the most important parameter to interact with the hydrometeors in clouds, convection intensity is another important parameter to reflect the ambient meteorological condition in clouds. So revealing the inner connection between the vertical distribution of hydrometeors and convection intensity is indispensable to make overall comprehension of TPV category.

\subsection{Comparison between Different Types of TPV Category} Cloud Clusters Based on the Difference of TBB on Cloudtop. The TBB on cloudtop is an important index for denoting the convective features of weather systems. In Section 3.1, the eastward-moving TPV category has been confirmed to be a typical mesoscale convective vortex (as shown in Figure 4); moreover, the TBB on cloudtop is an important index for the inversion of the precipitation [29], and it is extremely sensitive to the vertical structure of water vapor and convection intensity $[30,31]$. This paper adopted a similar classification criterion for the classification of TPV category cloud clusters based on the difference of TBB on cloudtop, proposed by $\mathrm{Fu}$ et al. [32]. The details of the classification 
criteria are as follows: (1) pure ice phase on cloudtop $(\mathrm{TBB} \leq 233 \mathrm{~K})$, (2) ice-water mixed-phase I on cloudtop $(233 \mathrm{~K}<\mathrm{TBB} \leq 253 \mathrm{~K})$, (3) ice-water mixed-phase II on cloudtop $(253 \mathrm{~K}<\mathrm{TBB}<273 \mathrm{~K})$, and (4) pure water phase at cloudtop (TBB $\geq 273 \mathrm{~K}$ ). However, considering that the TPV category cloud clusters usually possess severe convection feature and it dominates the cold cloud microphysics process, the fourth type of precipitation clouds (water phase on cloudtop) hardly appears practically. And the convection intensity is enhanced gradually during the eastward movement of the TPV category cloud clusters. Thus, this paper integrates the second type and third type into a whole one and removes the fourth type entirely. Furthermore, owing to the fact that the covered acreage spectrum of the TBB on cloudtop that satisfies different categories of TPV cloud clusters fluctuates widely, this paper prescribed that the pure ice phase type of the TPV category cloud clusters should satisfy not only the premise of the temperature threshold but also the covered acreage spectrum threshold of the TBB on cloudtop with reference to Maddox's criteria for MCSs [33] (Table 2); otherwise, the TPV category is classified into the ice-water mixed-phase of the TPV category cloud clusters.

This paper revealed the correspondence relationship between convection intensity and the vertical distribution of cloud hydrometers, as shown in Figure 11, when the convection intensity is strengthening (ice-water mixed-phase type shifting to pure ice phase type), the number concentration of ice crystals demonstrate an ascending tendency near the tropopause $(12 \mathrm{~km}-15 \mathrm{~km})$ in each key region, and the distribution of ice crystals presents a tendency of stretching upward. And the tendency of stretching upward for the distribution of ice crystals is also presented during the eastward movement of the TPV category. This discovery validates the previous conclusion that the convection of the TPV category shows an ascending tendency during eastward movement of the TPV category (Figure 4) otherwise. Besides, the feature of stratified distribution for ice crystals becomes prominent gradually with the TPV category cloud clusters moving eastward, especially in the MYR region. In addition, the average effective radius of ice crystals for the pure ice-phase type is larger than the ice-water mixed-phase type above $8 \mathrm{~km}$ height in each key region (Figure 12(a)), and the discrepancy just corresponds to the distinction between the two types of TPV cloud clusters in the number concentration.

The difference is also existing between the two types of TPV category cloud clusters in the cloud droplets, as shown in Figure 13, and when the convection intensity is strengthened (ice-water mixed-phase type shifting to pure ice phase type), the concentration of cloud droplets in the ice-water mixed layer (near $8 \mathrm{~km}$ ) presents a descending tendency. And the tendency of stretching downward for the distribution of cloud droplets is also presented during the eastward movement of TPV category, which is opposite to the tendency of ice crystals. In addition, the average effective radius of cloud droplets showing increasing tendency near the ground (the average terrain height within A1, A2, and A3 is different, and thus the baseline altitude of ground is
TABLE 2: The classification of TPV category cloud clusters based on the phase on cloudtop.

\begin{tabular}{lr}
\hline $\begin{array}{l}\text { Classification } \\
\text { criteria }\end{array}$ & $\begin{array}{c}\text { Range of threshold for TBB on } \\
\text { cloudtop and corresponding acreage }\end{array}$ \\
\hline Pure ice-phase type & (1) TBB $\leq 233 \mathrm{~K}$; (2) $S \geq 100000 \mathrm{~km}^{2}$ \\
\hline Ice-water mixed-phase type & $\begin{array}{r}\text { (1) } 233 \mathrm{~K}<\mathrm{TBB}<273 \mathrm{~K} ; \\
\text { (2) } S \geq 100000 \mathrm{~km}^{2}\end{array}$ \\
& (1) $\mathrm{TBB} \leq 233 \mathrm{~K} ;(2) S<100000 \mathrm{~km}^{2}$ \\
\hline
\end{tabular}

different) corresponds to each key region, as well as the decreasing tendency in the midair (Figure 12(b)). Similarly, the fragment and collision of cloud droplets likewise play a dominant role in the variation of the average effective radius.

\section{Conclusion and Discussion}

In order to make overall comprehension towards the cloud features of the TPV category cloud clusters, first 107 cases of eastward-moving TPV category that occurred in the summer half-year from 2007 to 2016 were picked out, and then this paper made further analysis of the clouds affiliated to this kind of weather system through several aspects, including the variation of frequency, convection intensity, precipitation distribution, the macroscopic feature of clouds, and the vertical distribution of cloud hydrometers in different classification criteria. The conclusions are as follows: the monthly variation of frequency for the eastward-moving TPV category reaches the peak point during May and June and falls down at the lowest point during July and September. In addition, the precipitation intensity and the convection intensity triggered by the eastward-moving TPV category cloud clusters showed an ascending tendency during the eastward movement. The eastward-moving TPV category cloud clusters is distinct from other clouds under the climatological background state, and the statistical results indicate that the proportion of the frequency of deep convection clouds within TPV category cloud clusters was much higher than other clouds under the climatological background state, especially in the lower level of troposphere, and the proportion of the frequency of multiple layer clouds and convective precipitation clouds within TPV category cloud clusters was higher than other clouds under climatological background state. The same tendency is likewise discovered during the eastward movement of TPV category cloud clusters. Obviously, the vertical distribution of different hydrometers exerts profound influence on the precipitation efficiency; hence, the concentration of ice crystals showed an ascending tendency in the middle and higher level of troposphere, and the concentration of cloud droplets also showed an ascending tendency in the ice-water mixed layer with the enhancement of precipitation intensity. The notable trend of stretching upward of the distribution of ice crystals and downward of the distribution of cloud droplets was demonstrated in the number concentration virtually during the eastward movement of the TPV category cloud clusters. The increasing tendency is also discovered in the effective average radius of the ice crystals and cloud droplets. The TBB on cloudtop is the direct parameter to 


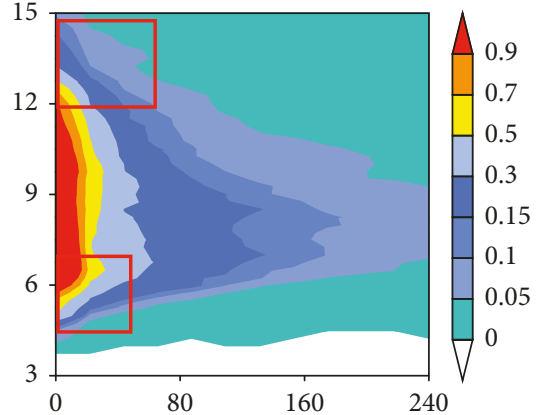

(a)

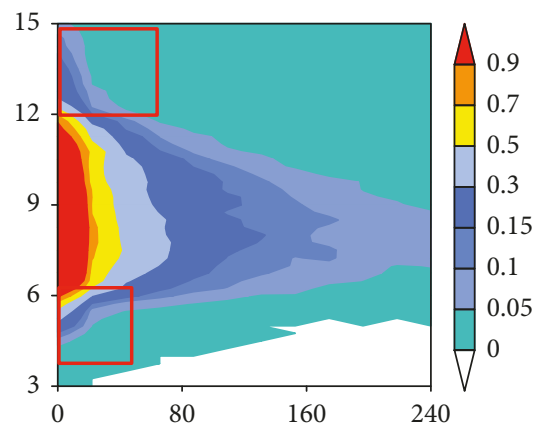

(d)

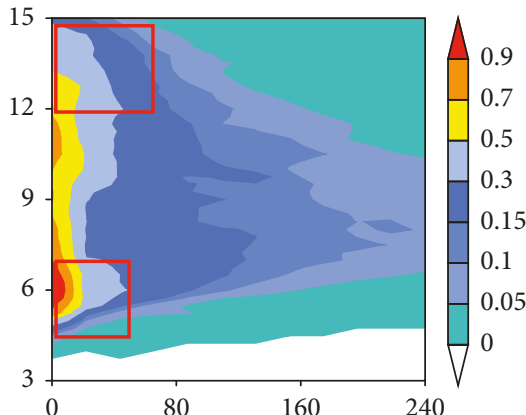

(b)

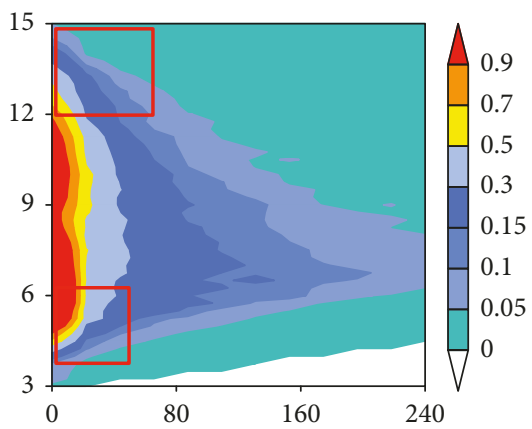

(e)

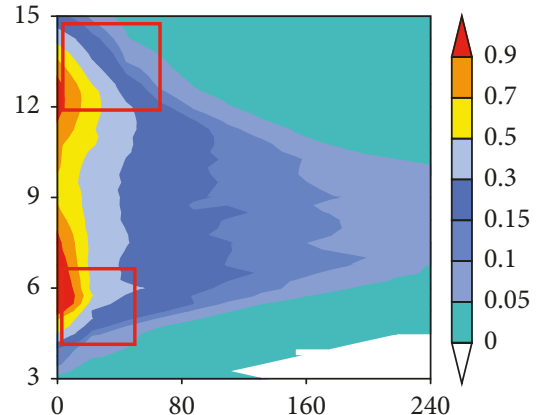

(c)

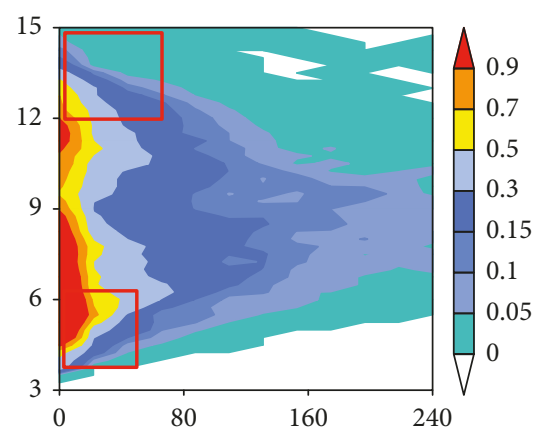

(f)

FIgURE 11: The vertical distribution of the number concentration of ice crystals corresponding to different types of TPV category cloud clusters over each key region: (a) iwc_TP (IC); (b) iwc_ESTP (IC); (c) iwc_MYR (IC); (d) iwc_TP (ILC); (e) iwc_ESTP (ILC); (f) iwc_MYR (ILC). ((a) TP, (b) ESTP, and (c) MYR represent the pure ice phase of the TPV category cloud clusters, and panels (d) TP, (e) ESTP. and (f) MYR represent the ice-water mixed-phase of TPV category; $X$-axis represents particles number concentration (unit: $\mathrm{mg} / \mathrm{m}^{3}$ ); $Y$-axis represents altitude (unit: $\mathrm{km}$ ); shaded color represents the percentage of frequency (unit: \%); and red and yellow box represents the significant discrepancy area)).

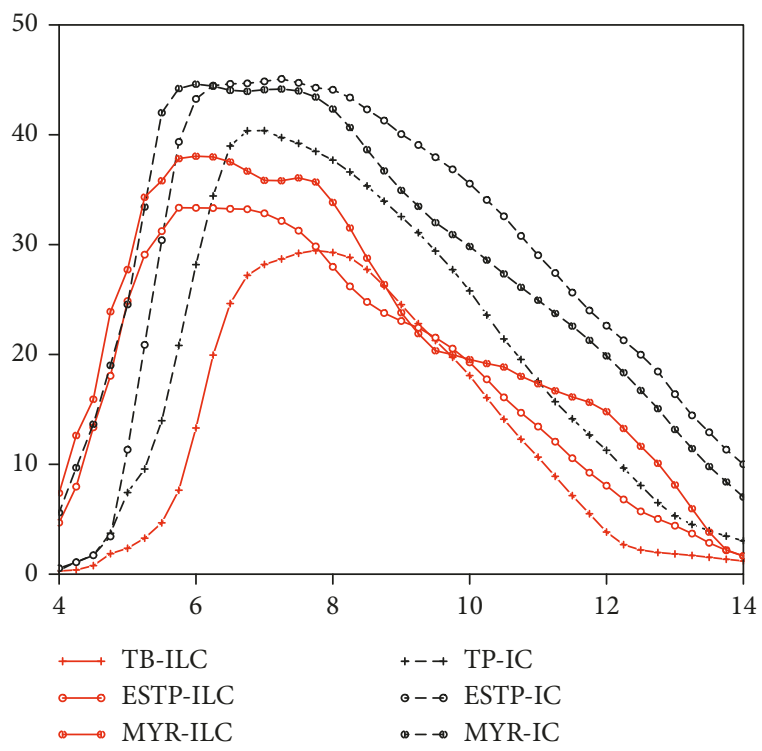

(a)

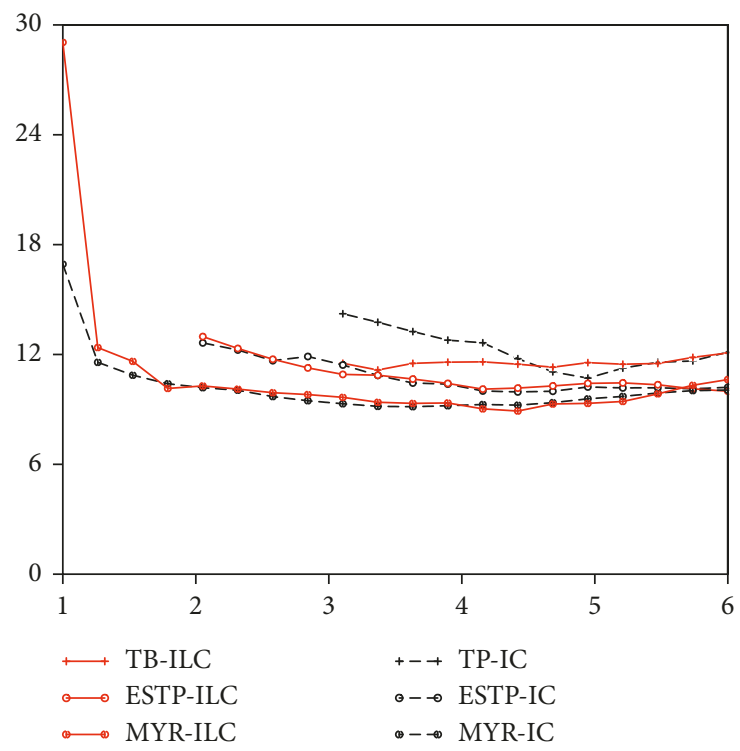

(b)

FIGURE 12: The vertical distribution of the average effective radius of different hydrometer particles corresponding to different types of TPV category cloud clusters in each key region: (a) effective radius of ice crystal particles; (b) effective radius of water-droplet particles ((a) ice crystals, (b) cloud droplets, the red legend represents the ice-water mixed-phase type, the black legend represents the pure ice phase type, $X$ axis represents altitude (unit: $\mathrm{km}$ ), and $Y$-axis represents average effective radius (unit: $\mu \mathrm{m})$ ). 


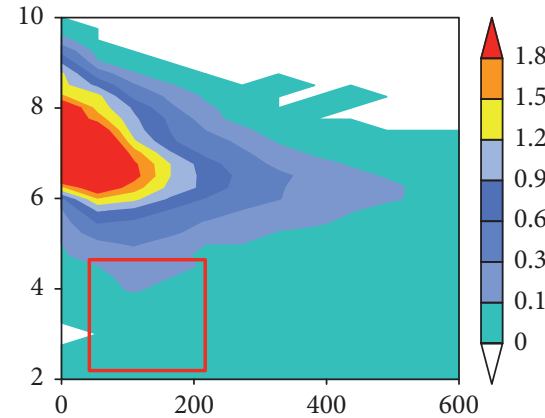

(a)

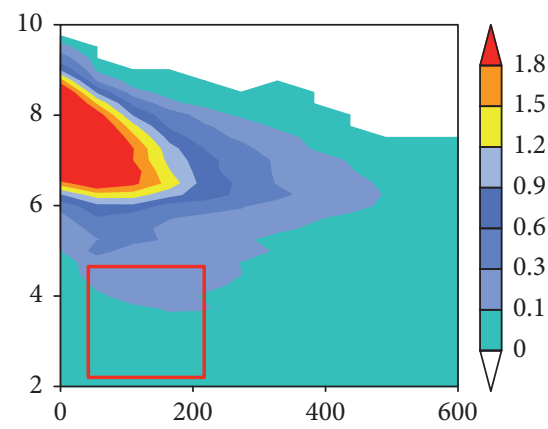

(d)

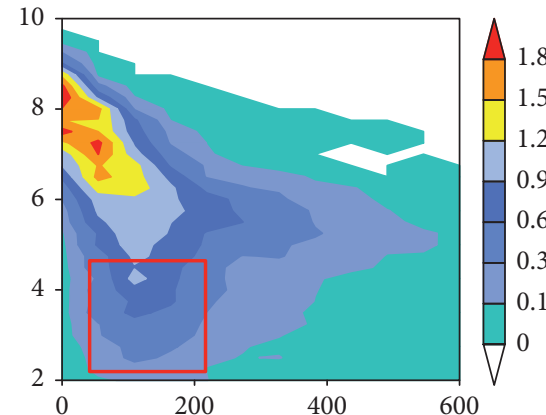

(b)

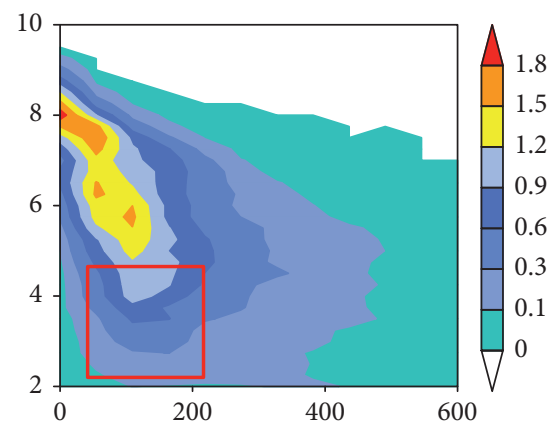

(e)

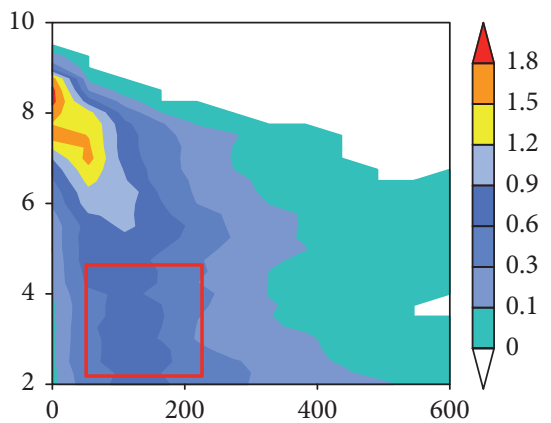

(c)

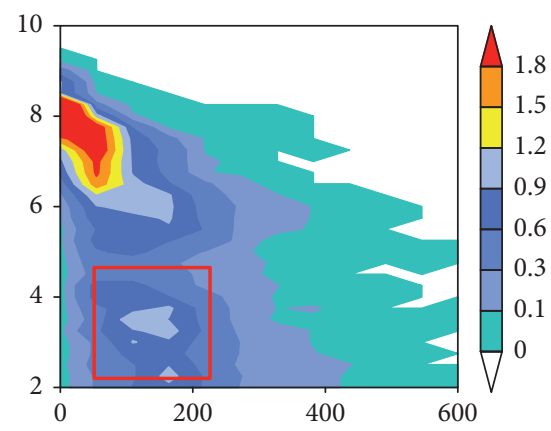

(f)

FIGURE 13: The vertical distribution of the number concentration of cloud droplets corresponding to different types of TPV category cloud clusters over each key region (same as above): (a) lwc_TP (IC); (b) lwc_ESTP (IC); (c) lwc_MYR (IC); (d) lwc_TP (ILC); (e) lwc_ESTP (ILC); (f) lwc_MYR (ILC).

describe the convection intensity, and the concentration of ice crystals showed an ascending tendency at the tropopause, but the concentration of cloud droplets showed the opposite tendency in ice-water mixed layer with the enhancement of convection intensity. The discipline that stretching upward of the distribution of ice crystals and downward of the distribution of cloud droplets is the same as the former classification criterion based on the difference of precipitation intensity, because the precipitation intensity and convection intensity truly strengthened during the eastward movement of the TPV category cloud clusters. Generally speaking, the conclusions drawn from the classification criterion based on the cloudtop phase share some similarities with that one based on precipitation intensity. Actually, these two parameters as two notable properties of the cloud clusters are closely related with each other. Despite some commonness achieved from these two different classification criteria, the discrepancies also exist practically. For example, when TPV category cloud clusters move eastward in the downstream of the TP area, the average effective radius of cloud droplets presents opposite variation tendency shown in these two different classification criteria. In spite of a significantly positive correlation between convection intensity and precipitation intensity in the cloud clusters [34], the discrepancies discussed above indicate that convection intensity and precipitation intensity are not the only dominant factors to determine the distribution of cloud hydrometers possibly; in other words, although the three ones are associated and interacting mutually, the closed function relationship among them cannot be established, and other meteorology factors related to the ambient condition should also be taken into account.

This paper has partially revealed cloud features of one typical cloud defined as the eastward-moving TPV category cloud clusters. Although some significant conclusions have been drawn, a few defects is retained as follows: above all, the accuracy of CloudSat data is affected by data bias especially in the complex terrain region, it is essential to further revise the results and methods presented herein until satellite remote sensing technology is improved in future. Besides, as for the conclusions depended on the different classification criteria based on the difference of precipitation intensity and convection intensity, it is maybe appropriate to adjust the scope of classification criteria, in order to conform with the local climate characteristics, but the exact scope of classification criteria of precipitation intensity and convection intensity over the three different key regions needs further investigation. Finally, his paper just provided statistical results of cloud features for the eastward-moving TPV category cloud clusters, and it hardly compares and analyzes the disparities of the evolution mechanism among different typical cases of eastward-moving TPV category cloud clusters, but the mechanism plays an extremely important role of the comprehension of inducement of heavy rainstorms triggered by the eastward-moving TPV category cloud clusters in the downstream of Tibetan Plateau. As a result, it is essential to carry out further investigations on these scientific issues in future. 


\section{Data Availability}

The Cloudsat data were obtained from http://cloudsat.atmos. colostate.edu/. The ERA-interim reanalysis data are available online https://www.ecmwf.int/en/forecasts/datasets. The FY satellite data were provided by National Satellite Meteorological Centre from http://satellite.nsmc.org.cn/portalsite/ default.aspx. The CMORPH hourly precipitation data were accessed from http://data.cma.cn/search/keywords.html.

\section{Conflicts of Interest}

The authors declare that they have no conflicts of interest.

\section{Acknowledgments}

This work was supported by the National Natural Science Foundation of China (Grant nos. 41620104009, 91637211, 2018YFC1507200, 41375057), The National Key R\&D Program of China (Grant no. 2018YFC1507200) and the Science and Technology Foundation of HuBei Meteorological Bureau (Grant no. 2017Y03).

\section{References}

[1] F. Wang, S. Yang, and T. W. Wu, "Radiation budget biases in AMIP5 models over the East Asian monsoon region," Journal of Geophysical Research: Atmospheres, vol. 119, no. 23, pp. 13400-13426, 2014.

[2] G. Chen and G. P. Li, "Characteristics of tangential flow field of the Tibetan Plateau vortices and associated waves," Acta Meteorologica Sinica, vol. 69, no. 6, pp. 956-963, 2011.

[3] S. H. Yu and W. L. Gao, "Observational analysis on the movement of vorticles before or after moving out the Tibetan Plateau," Acta Meteorological Sinica, vol. 64, no. 3, pp. 392399, 2006.

[4] S. H. Yu, W. L. Gao, and J. Peng, "Statistical analysis on influence of Tibetan Plateau Vortex activity on precipitation in China," Chinese Journal of Plateau Meteorology, vol. 31, no. 3, pp. 592-604, 2012.

[5] D. Z. Ye and Y. X. Gao, The Meteorology of Tibetan Plateau, The Science Publishing Press, Beijing, China, 1979.

[6] S. H. Yu and W. L. Gao, "The large-scale conditions of the vortex moving out of Tibetan Plateau," Chinese Journal of Plateau Meteorology, vol. 27, no. 6, pp. 1276-1287, 2008.

[7] X. L. Zhang, S. Y. Tao, and S. L. Zhang, "Three types of heavy rainstorms associated with the Meiyu front," Chinese Journal of Atmospheric Sciences, vol. 28, no. 2, pp. 187-205, 2004.

[8] C. X. Shi, J. X. Jiang, and Z. Y. Fang, "A study on the features of severe convective cloud clusters causing serious flooding over Changjiang River Basin in 1998," Chinese Journal of Climate and Environmental Research,vol. 5, no. 3, pp. 279286, 2000.

[9] G. Zhou, X. D. Xu, and L. S. Chen, "Convective clouds and mesoscale convective system over the Tibetan Plateau in summer," Chinese Journal of Applied Meteorology Science, vol. 13, no. 4, pp. 448-456, 2002.

[10] J. Y. Wu, "The comparison and analysis of detection mode based on Ka wave band transmitter onboarding millimeter wave radar and its application on in the Tibetan Plateau Vortex," Master dissertation, Chinese Academy of Meteorological Sciences, Beijing, China, 2016.
[11] Y. G. Zheng, J. Chen, and P. J. Zhu, "The distribution of mesoscale convective system and daily variation characteristics," Chinese Science Bulletin, vol. 53, no. 4, pp. 471-481, 2008.

[12] B. Y. Yang, H. Zhang, and J. Peng, "Analysis on global distribution characteristics of cloud microphysical and optical properties based on the CloudSat data," Chinese Journal of Plateau Meteorology, vol. 33, no. 4, pp. 1105-1118, 2014.

[13] X. Zhang, K. Q. Duan, and P. H. Shi, "Cloud vertical profiles from CloudSat data over the eastern Tibetan Plateau during summer," Chinese Journal of Atmospheric Sciences, vol. 39, no. 6, pp. 1073-1080, 2015.

[14] H. Wang, Y. L. Luo, and R. H. Zhang, "Analyzing seasonal variation of clouds over the Asian monsoon regions and the Tibetan Plateau region using CloudSat/CALIPSO data," Chinese Journal of Atmospheric Sciences, vol. 35, no. 6, pp. 1118-1131, 2011.

[15] J. J. Liu and B. D. Chen, "Cloud occurrence frequency and structure over the Qinghai-Tibetan Plateau from CloudSat observation," Chinese Journal of Plateau Meteorology, vol. 36, no. 3, pp. 632-642, 2017.

[16] D. S. Yang and P. C. Wang, "Tempo-spatial distribution characteristics of cloud particles size over China during summer," Chinese Journal of Climate and Environmental Research, vol. 17, no. 4, pp. 433-443, 2012.

[17] C. H. Shi, H. Li, B. Zheng, G. Dong, and L. R. Qiang, "Stratosphere-troposphere exchange corresponding to a deep convection in warm sector and abnormal subtropical front induced by a cutoff low over East Asia," Chinese Journal of Geophysics, vol. 57, no. 1, pp. 21-30, 2014.

[18] Z. M. Chen, W. B. Min, Q. Liao et al., "A case study on coupling interaction between Plateau and Southwest Vortexes," Chinese Journal of Plateau Meteorology, vol. 23, no. 1, pp. 75-80, 2004.

[19] H. W. Barker, A. V. Korolev, D. R. Hudak et al., "A comparison between CloudSat and aircraft data for a multilayer, mixed phase cloud system during the Canadian CloudSatCALIPSO validation project," Journal of Geophysical Research, vol. 113, 2008

[20] Y. Qiu, T. Choularton, J. Crosier, and Z. Liu, "Comparison of cloud properties between CloudSat retrievals and airplane measurements in mixed-phase cloud layers of weak convective and stratus clouds," Advances in Atmospheric Sciences, vol. 32, no. 12, pp. 1628-1638, 2015.

[21] X. Feng, C. Liu, R. Rasmussen, and G. Fan, "A 10-yr climatology of Tibetan Plateau vortices with NCEP climate forecast system reanalysis," Journal of Applied Meteorology and Climatology, vol. 53, no. 1, pp. 34-46, 2014.

[22] R. Zhong, L. H. Zhong, L. J. Hua et al., "A climatology of Southwest vortex during 1979-2008," Journal of Atmospheric and Oceanic Science Letters, vol. 7, no. 6, pp. 577-583, 2014.

[23] S. H. Yu, W. L. Gao, and J. Peng, "Circulation features of sustained departure plateau vortex at middle tropospheric level," Chinese Journal of Plateau Meteorology, vol. 34, no. 6, pp. 1540-1555, 2015.

[24] S. H. Yu, W. L. Gao, and J. Peng, "Circulation features of sustained departure plateau vortex at upper tropospheric level," Chinese Journal of Plateau Meteorology, vol. 35, no. 6, pp. 1441-1455, 2016.

[25] Y. Gao, "Analysis and numerical simulation on the vertical structure and microphysics feature of precipitation cloud and the latitude zone of Tibetan Plateau over China," Dissertation, 
University of Chinese Academy of Science, Beijing, China, 2014.

[26] X. L. Guo, D. H. Fu, and Z. X. Hu, "Progress in cloud physics, precipitation, and weather modification during 2008-2012," Chinese Journal of Atmospheric Sciences, vol. 37, no. 2, pp. 351-363, 2013.

[27] Y. Huang and X. Cui, "Dominant cloud microphysical processes of a torrential rainfall event in Sichuan, China," Advances in Atmospheric Sciences, vol. 32, no. 3, pp. 389-400, 2015.

[28] X. Cui, Y. Zhou, and X. Li, "Cloud microphysical properties in tropical convective and stratiform regions," Meteorology and Atmospheric Physics, vol. 98, no. 1-2, pp. 1-11, 2007.

[29] H. Iwabuchi, M. Saito, Y. Tokoro et al., "Retrieval of radiative and microphysical properties of clouds from multispectral infrared measurements," Progress in Earth and Planetary Science, vol. 3, no. 1, pp. 3-32, 2016.

[30] R. Fulton and G. M. Heymsfield, "Microphysical and radiative characteristics of convective clouds during COHMEX," Journal of Applied Meteorology, vol. 30, no. 1, pp. 98-116, 1991.

[31] R. F. Adler, H.-Y. M. Yeh, N. Prasad, W.-K. Tao, and J. Simpson, "Microwave simulations of a tropical rainfall system with a three-dimensional cloud model," Journal of Applied Meteorology, vol. 30, no. 7, pp. 924-953, 1991.

[32] Y.F. Fu, X. Pan, and G. S. Liu, "Characteristics of precipitation based on cloud brightness temperature and storm tops in summer Tibetan Plateau," Chinese Journal of Atmospheric Sciences, vol. 40, no. 1, pp. 102-120, 2016.

[33] A. M. Robert, "Mesoscale convective complexes," Bulletin of the American Meteorological Society, vol. 61, no. 11, pp. 1374-1387, 1980.

[34] X. T. Liu, "Cloud parameters retrieval and precipitation clouds identification over global tropic based on TRMM spectral measurements," Dissertation, University of Science and Technology of China, Hefei, China, 2012. 

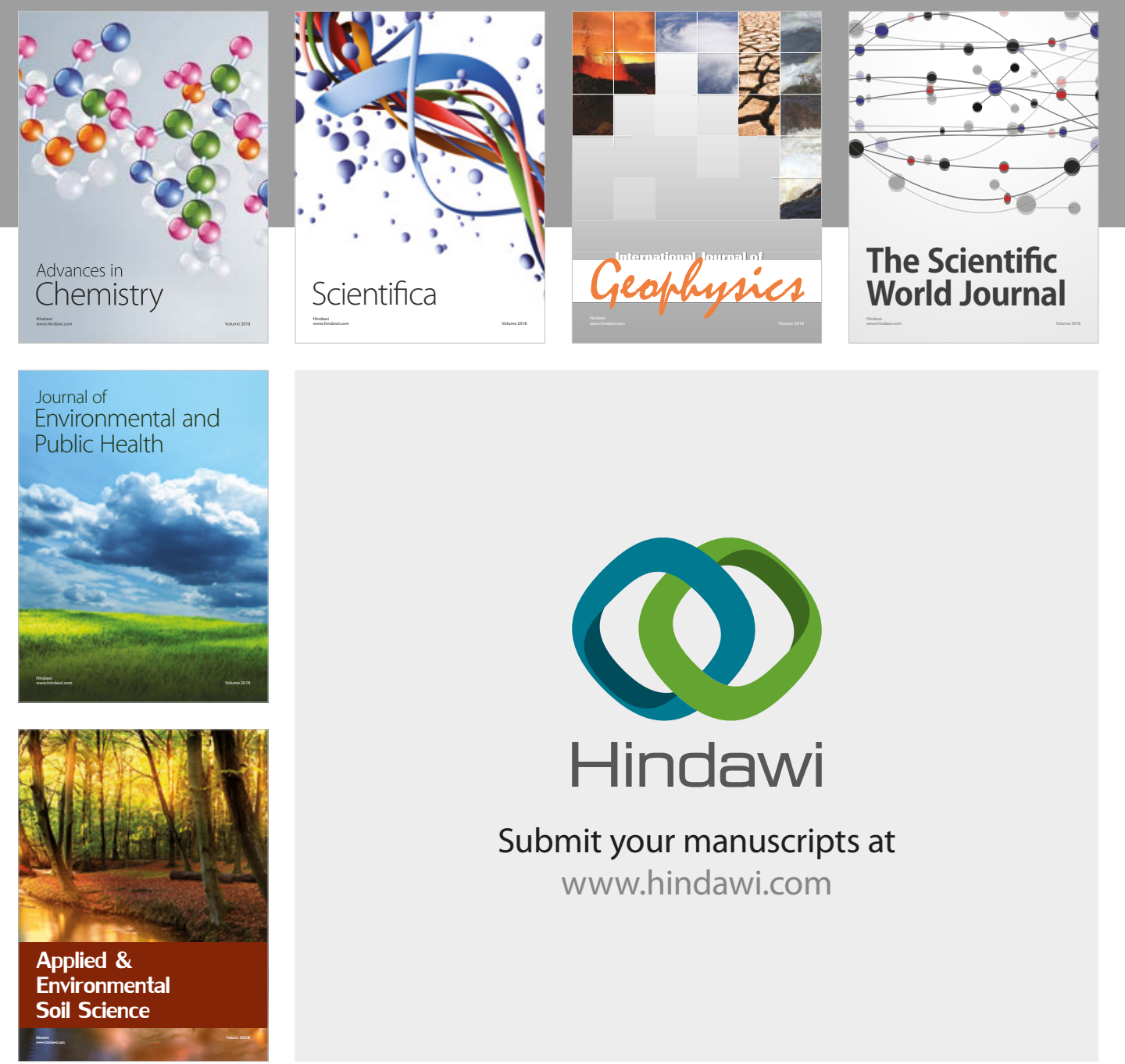

The Scientific

\section{World Journal}
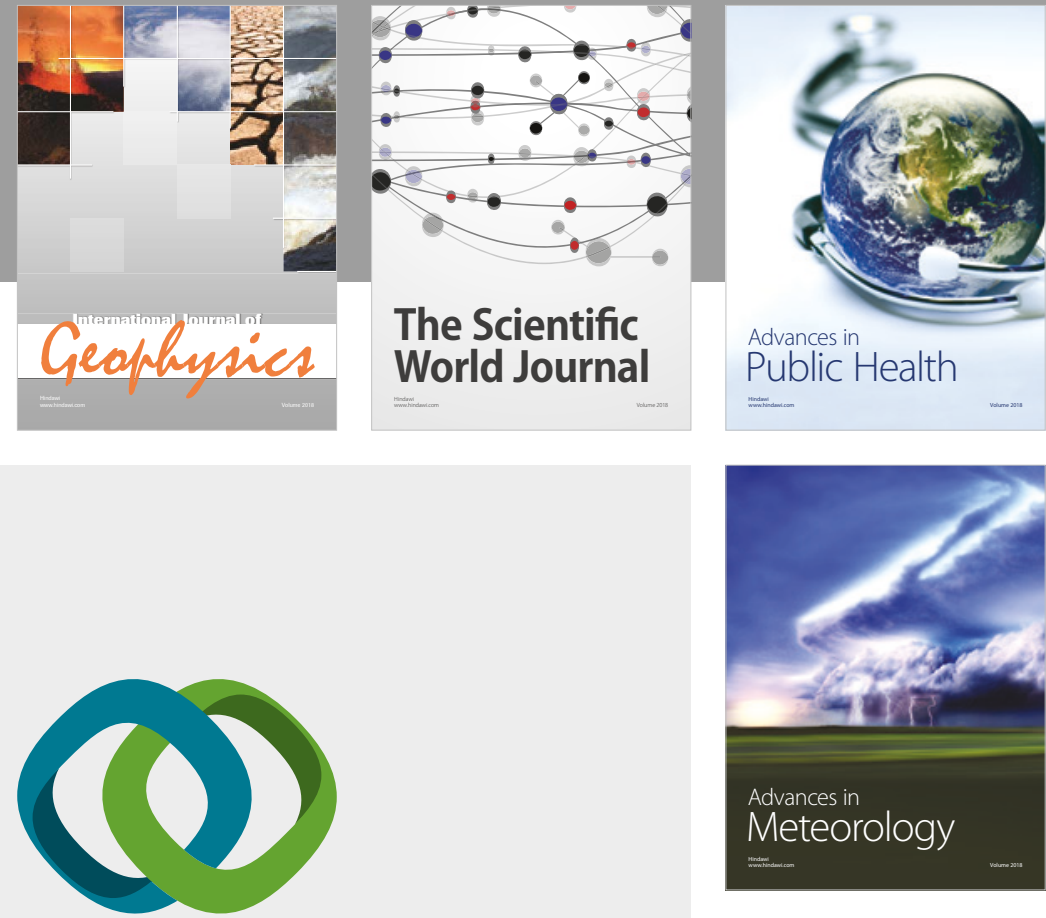

Advan

Public Health

\section{Hindawi}

Submit your manuscripts at

www.hindawi.com
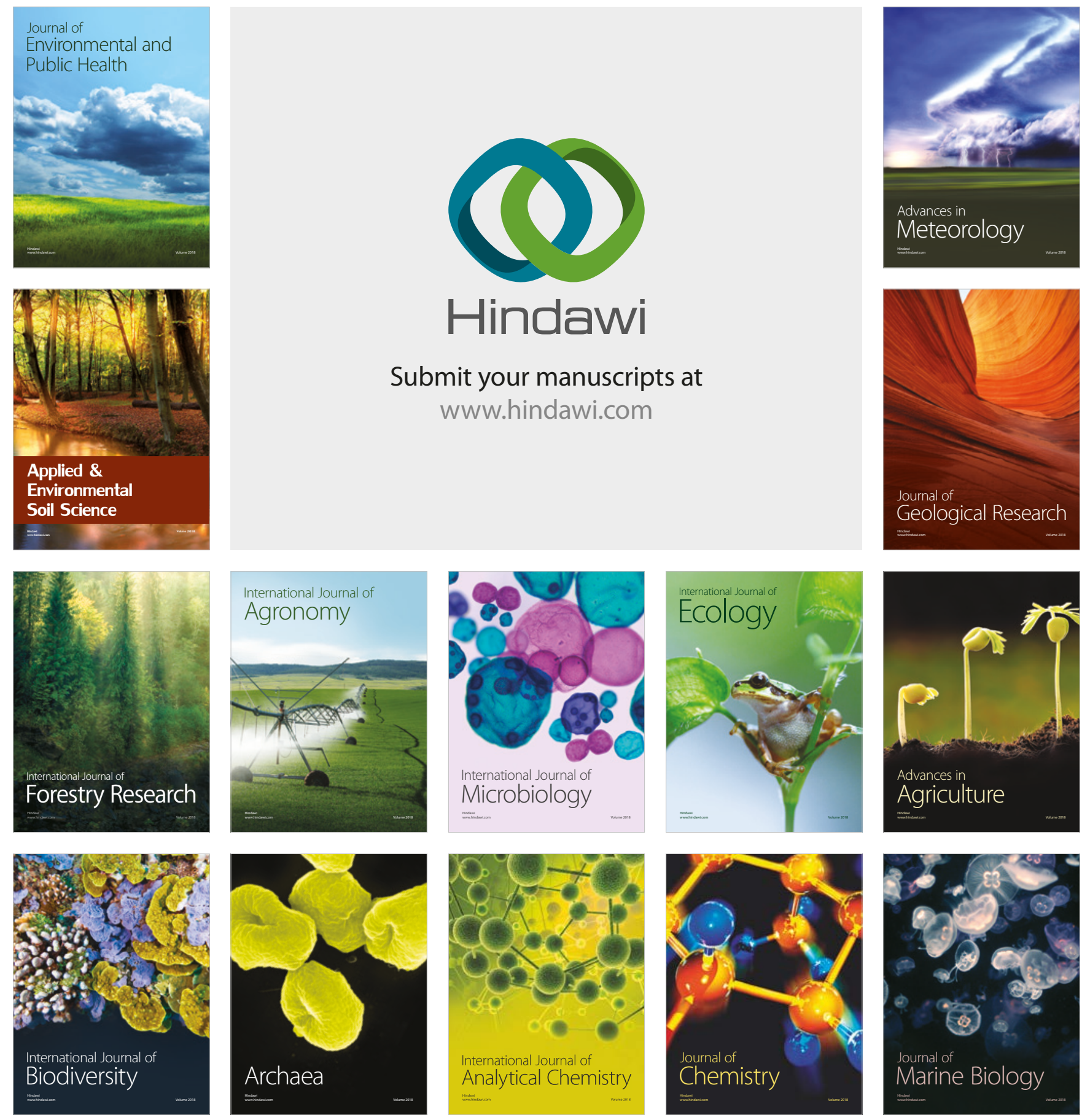